\title{
Jiří Tůma
}

Some finite congruence lattices, I

Czechoslovak Mathematical Journal, Vol. 36 (1986), No. 2, 298-330

Persistent URL: http://dml.cz/dmlcz/102093

\section{Terms of use:}

(C) Institute of Mathematics AS CR, 1986

Institute of Mathematics of the Czech Academy of Sciences provides access to digitized documents strictly for personal use. Each copy of any part of this document must contain these Terms of use.

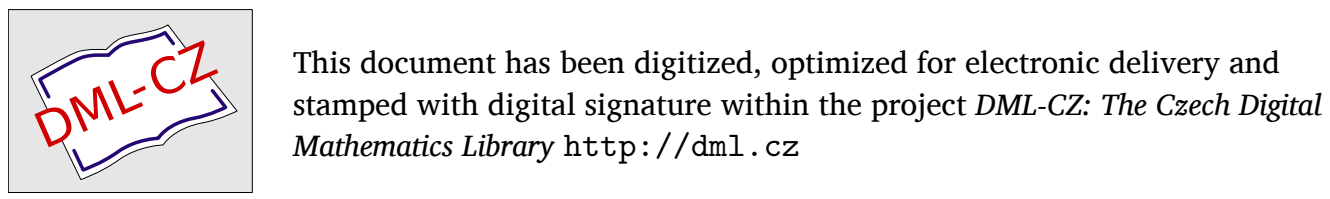




\title{
SOME FINITE CONGRUENCE LATTICES, I
}

\author{
Jiří TŮma, Praha
}

(Received January 2, 1985)

Introduction. The paper deals with the finite congruence lattice representation problem and contains examples of finite permutational algebras with congruence lattices isomorphic to partition, Boolean and quasi-ordering lattices.

The problem whether every finite lattice is isomorphic to the congruence lattice of a finite algebra has attracted some attention in the last ten years. Results obtained so far have turned the attention to essentially unary algebras, and among them especially to groups of permutations. A finite unary algebra whose set of operations is a transitive group of permutations is called here a permutational algebra. The congruence lattice of a permutational algebra $(X, G)$ is known to be isomorphic to the interval $\left[S_{x}, G\right]$ in the subgroup lattice of $G$ between the stabilizer of a point $x \in X$ and the whole group $G$ (see [7]). It follows that by constructing a permutational algebra with a given congruence lattice we simultaneously find a finite group containing the given lattice as an interval in its subgroup lattice. Hence our paper could be also titled "Some intervals in subgroup lattices of finite groups".

Our interest in permutational algebtas does not mean any restriction in the finite congruence lattice representation problem, since by a result of [7], every finite lattice is isomorphic to the congruence lattice of a finite algebra iff every finite lattice is isomorphic to an interval in the subgroup lattice of a finite group. This equivalent formulation is sometimes considered to suggest the negative answer. One of the consequences of this feeling is that not too many lattices were really proved to be isomorphic to finite congruence lattices. Up to now, the largest class of finite congruence lattices closed under finite products, homomorphic images and sublattices is the class of finitely fermentable lattices described in [9]. This class contains all distributive lattices. Obviously, every finite partition lattice is the congruence lattice of a finite algebra - no operations are needed. Further, we have infinitely many congruence lattices of length two (not all, now $M_{13}$ is the smallest undecided case), and some other examples, such as McKenzie's example of a finite congruence lattice which is not isomorphic to the congruence lattice of any finite algebra with one operation ([4]). Moreover, an unpublished result of the author shows that the class of finite congruence lattices is closed under (finite) products. 
In view of the equivalent formulation of the finite congruence lattice representation problem quoted above, it is also of interest to examine which lattices are known to be isomorphic to congruence lattices of permutational algebras. Here the situation is even worse. From the infinite classes of finite congruence lattices listed above only the examples of length two are obtained as congruences of permutational algebras. Known constructions of finitely fermentable and partition lattices as congruence lattices do not use transitive groups of permutations.

Representations of some finite lattices as congruence lattices of permutational algebras are the main topic of this paper. In Section 1 we present a representation of partition lattices. In other words, we prove that partition lattices are isomorphic to intervals in subgroup lattices of finite symmetric groups. Section 2 contains a representation of Boolean lattices as intervals in collineation groups of finite projective spaces. This is a known result in group theory. The last Section 4 contains a representation of quasi-ordering lattices as congruence lattices of permutational algebras. The group is again the collineation group of a finite projective space. The representation of quasi-ordering lattices and intervals in them as finite congruence lattices is the main new result of this paper. As a corollary we get also a representation of finite distributive lattices by congruences of permutational algebras, since any finite distributive lattice is isomorphic to an interval in a quasi-ordering lattice. In the same way we obtain several new representations of Boolean lattices. This is proved together with some auxiliary results on quasi-ordering lattices in the short Section 3. To characterize intervals in quasi-ordering lattices is an interesting unsolved problem related to the present work.

Although formulated as independent constructions, the algebras studied in this paper have many common properties. Roughly speaking, they are constructed by amalgamating the Cayley representation of a symmetric group. Below we state some axioms to specify what we mean by "good amalgams". First of all we introduce the concept of an induced permutational algebra. Take a permutation group $(X, G)$ and a set $A \subseteq X$. By $\mathrm{Stab}_{A}$ we denote the subgroup of $G$ containing all $g \in G$ such that $g(A)=A$, and by PStab $_{A}$ the pointwise stabilizer of $A-$ i.e. the set of all $g \in G$ such that $g(x)=x$ for all $x \in A$. If $g, h \in \mathrm{Stab}_{A}$ belong to the same coset of $\mathrm{PStab}_{A}$, then the restrictions of $g$ and $h$ to $A$ coincide. It follows that we may regard the group $\mathrm{Stab}_{A} / \mathrm{PStab}_{A}$ as a group of permutations on $A$, and we call the algebra $\left(A, \operatorname{Stab}_{A}\right.$ : $: \mathrm{PStab}_{A}$ ) the induced permutational algebra (or induced permutation group) on $A$. Now we are ready to define "good amalgams".

A permutational algebra $(X, G)$ together with a collection $\mathscr{A}$ of subsets of $X$ (called apartements) is a permutational algebra (or permutation group) of type $A_{n}$ if it satisfies the following conditions:

(i) (connectedness) for any $x, y \in X$ there is a sequence of apartements $A_{0}, A_{1}, \ldots$ $\ldots, A_{k}$ such that $x \in A_{0}, y \in A_{k}$ and $A_{i} \cap A_{i+1}$ is non-empty for all $i=0,1, \ldots, k-1$;

(ii) (induced algebras) for all $A \in \mathscr{A}$, the induced group on $A$ is isomorphic to the Cayley representation of the symmetric group on $n+1$ letters; 
(iii) (transitivity) the group $G$ acts transitively on the set of all incident pairs $(x, A)$, where $x \in X, A \in \mathscr{A}$ and $x \in A$.

The symbol $A_{n}$ refers to the classification of finite Coxeter groups - see [2].

The shape of the congruence lattice of a permutational algebra of type $A_{n}$ is influenced by intersections of apartements. This will be studied in the second part of this paper together with some other examples, their apartement structures and general methods of constructing such algebras.

Our axioms resemble in some directions the axioms and properties of algebras with tame minimal sets, the first concept of tame congruence theory developed by $\mathrm{R}$. McKenzie in [4] and surveyed in [5] and [6]. In fact, the concept of algebras with tame minimal sets and the concept of buildings due to J. Tits ([3], [10], [11]) were the main inspiration for the present work.

We use only standard concepts, although our notation is sometimes unconventional. Necessary definitions are stated throughout the text. Here we point out only that a complete lattice homomorphism is a homomorphism preserving also the least and the greatest elements. By $X \subset Y$ we mean always proper inclusion (hence $X \neq Y$ ). The partition, Boolean and quasi-ordering lattices on a set $I$ are denoted respectively by $\Pi(I), B(I)$ and $\Omega(I)$. Finally, all the structures throughout the text are finite.

P. Pálfy informed me that representations of distributive lattices as congruence lattices of permutational algebras follow also from a result of Silcock [13]. I am also indebted to P. Pudlák for making several suggestions improving the final version of the paper.

1. Representations of partition lattices. In this section we assume $I=\{0,1, \ldots$ $\ldots, n-1\}$. Let us take a vector of positive integers $p=\left(p_{0}, p_{1}, \ldots, p_{n-1}\right)$ with the following property

$$
\text { for any two sets } J, K \subseteq I, \quad \sum_{J} p_{j}=\sum_{K} p_{k} \text { iff } J=K .
$$

Vectors $p$ with this property exist, one can take e.g. $p_{i}=2^{i}$ for all $i \in I$.

Now take a set $X$ of cardinality $N=\sum_{i=0}^{n-1} p_{i}$. By a $p$-partition of $X$ we mean a complete lattice embedding $e: B(I) \rightarrow B(X)$ such that $e(i)$ has cardinality $p_{i}$. Then we have

$$
e^{\prime}(J)=\bigcup_{j \in J} e^{\prime}(j) \text { for all } J \subseteq I .
$$

Since $|X|=\left|e^{\prime}(I)\right|=\sum_{i \in I}\left|e^{\prime}(i)\right|$, all the sets $e^{(}(i)$ are mutually disjoint, and $\left.\left\{e^{\prime} i\right): i \in I\right\}$ is a partition of $X$ of type $p$. Conversely, any partition $\{e(i): i \in I\}$ of $X$ of type $p$ can be extended to a $p$-partition $e$ by (1.2). Since all the cardinalities $p_{i}$ are mutually different, the correspondence is one-to-one. This explains our terminology.

Let us denote by $E$ the set of all $p$-partitions of $X$. If $\varphi$ is a permutation of $X$ and $e$ a $p$-partition of $X$, then $\varphi \circ e$ is also a $p$-partition. It follows that the symmetric group $\operatorname{Sym}(X)$ acts as a group of permutations of $E$ and this action is obviously 
transitive. We denote the action of a permutation $\varphi \in \operatorname{Sym}(X)$ on the set $E$ by the same symbol $\varphi$; it will not lead to any confusion. The action of $\operatorname{Sym}(X)$ on $E$ will be denoted by $G$. We shall prove that the congruence lattice $\operatorname{Con}(E, G)$ is isomorphic to the partition lattice $\Pi(I)$.

To this end we define a mapping $Z: \Pi(I) \rightarrow \Pi(E)$ as follows:

$$
\left.(e, f) \in Z(\pi) \text { iff } e_{(}^{\prime} J\right)=f^{\prime}(J) \text { for all blocks } J \text { of } \pi \text {. }
$$

Theorem 1A. The mapping $Z: \Pi(I) \rightarrow \Pi(E)$ defined by $(1.3)$ is a complete lattice embedding.

Proof. We prove that $Z=\Lambda \circ S \circ v$, where $\Lambda, v$ are lattice isomorphisms and $S$ is a complete lattice embedding.

Take an arbitrary partition $\omega \in \Pi(X)$ of type $p$ and denote its blocks by $Y_{0}, Y_{1}, \ldots$ $\ldots, Y_{n-1},\left|Y_{i}\right|=p_{i}$. The upper interval $\left[\omega, \iota_{X}\right]$ in $\Pi(X)$ is isomorphic to $\Pi(I)$, and we denote by $v$ the obvious isomorphism assigning to each $\pi \in \Pi(I)$ the partition $\tilde{\pi} \in \Pi(X)$ with blocks $\left\{\bigcup_{j \in J} Y_{j}: J \in \pi\right\}$.

Next we take a mapping $S: \Pi(X) \rightarrow \Pi(\operatorname{Sym}(X))$ defined by $(\varphi, \psi) \in S(\delta)$ iff $\varphi(Y)=\psi(Y)$ for all blocks $Y \in \delta$. This mapping is known to be a complete lattice embedding (see [1] or [12]).

Finally, we consider a mapping $\Lambda$ : $\operatorname{Sym}(X) \rightarrow \Pi(X)$ defined by $\Lambda(\varphi)=\left\{\varphi\left(Y_{i}\right)\right.$ : $i \in I\}$. This mapping is not injective, and we have $\Lambda(\varphi)=\Lambda(\psi)$ iff $\varphi\left(Y_{i}\right)=\psi\left(Y_{i}\right)$ for all $i \in I$, hence $\Lambda(\varphi)=\Lambda(\psi)$ iff $(\varphi, \psi) \in S(\omega)$. Since any partition of $X$ of type $p$ is of the form $\Lambda(\varphi)$ for some $\varphi$, the mapping $\Lambda$ establishes a bijection between blocks of the partition $S(\omega)$ and partitions of $X$ of type $p$. This bijection induces an isomorphism $\left.\Lambda: \Pi\left(\operatorname{Sym}^{\prime} X\right)\right) \rightarrow \Pi(E)$.

To prove $Z=\Lambda \circ S \circ v$ take two $p$-partitions $e, f \in E$ and find permutations $\varphi, \psi \in \operatorname{Sym}(X)$ such that $\Lambda(\varphi)=\{e(i): i \in I\}$ and $\Lambda(\psi)=\{f(i): i \in I\}$. By the definitions we have $(e, f) \in Z(\pi)$ iff $(\varphi, \psi) \in S(\tilde{\pi})$, hence $Z=\Lambda \circ S \circ v$.

Theorem 1B. The congruence lattice $\operatorname{Con}(E, G)$ is isomorphic to $\Pi(I)$.

Proof. We show that $\operatorname{Con}(E, G)=\operatorname{Im}(Z)$. A simple observation gives $\operatorname{Im}(Z) \subseteq$ $\subseteq \operatorname{Con}(E, G)$. Indeed, take $(e, f) \in Z(\pi)$ and $\varphi \in \operatorname{Sym}(X)$. Then $\left.e^{\prime} J\right)=f(J)$ for all blocks $J$ of $\pi$, hence $\varphi e(J)=\varphi f(J)$ for all $J \in \pi$, therefore $(\varphi e, \varphi f) \in Z(\pi)$. It remains to prove the converse inclusion $\operatorname{Con}(E, G) \subseteq \operatorname{Im}(Z)$. It will be proved by an induction on $\pi$ in the lattice $\Pi(I)$.

Take two $p$-partitions $e, f \in E$. By $\pi(e, f)$ we denote the least partition $\pi \in \Pi(I)$ such that $(e, f) \in Z(\pi)$, and by $\operatorname{Con}(e, f)$ the least congruence relation of $(E, G)$ containing the couple $(e, f)$. We want to prove $\operatorname{Con}(e, f)=Z(\pi(e, f))$. This is obvious if $\pi(e, f)$ is the least element of $\Pi(I)$. Now suppose that $\pi=\pi(e, f)$ is an atom in $\Pi(I)$. It has just one non-trivial block $\{i, j\}$. Then we have $\left.e^{\prime}\{i, j\}\right)=f(\{i, j\})$ and $\left.e_{(}^{\prime} k\right)=f(k)$ for $k \neq i, j$. Moreover, $e \neq f$, hence $e_{(i)} \neq f(i)$ and $e_{(j)} \neq f(j)$.

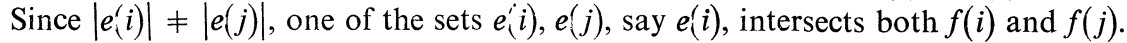
Take arbitrary elements $x \in e^{\prime}(i) \cap f(i)$ and $y \in e(i) \cap f(j)$. Then $x \neq y$. Consider 
the transposition $\varphi=(x, y)$. Since $x, y$ are in the same block of $e$, we get $\varphi e=e$. On the other hand, $x, y$ are in different blocks of $f$, hence $\varphi f \neq f$. It follows that $(e, \varphi f) \in \operatorname{Con}(e, f)$, hence also $(f, \varphi f) \in \operatorname{Con}(e, f)$. Moreover, we have that the symmetric difference $f(i)-\varphi f(i)=f(j)-\varphi f(j)=\{x, y\}$.

If $(g, h) \in Z(\pi)$, we can find a sequence $g(i)=X_{0}, X_{1}, \ldots, X_{m}=h(i)$ of subsets of $g(\{i, j\})=h(\{i, j\})$ such that the symmetric difference of any two subsequent sets has cardinality two.

Now for any $l=0,1, \ldots, m-1$ take a permutation $\varphi_{l} \in \operatorname{Sym}(X)$ sending $f(i)$ to $X_{1}, \varphi f(i)$ to $X_{l+1}, f(\{i, j\})$ to $g(\{i, j\})$ and $f(k)$ to $g(k)$ for all $k \neq i, j$. Since $|f(i)-\varphi f(i)|=\left|Y_{1}-Y_{l+1}\right|$, such a permutation $\varphi_{l}$ exists.

We have $\varphi_{l+1} f=\varphi_{l} \varphi f$ for all $l=0,1, \ldots, m-1, \varphi_{0} f=g$ and $\varphi_{m-1} \varphi f=h$. In the sequence $g=\varphi_{0} f, \varphi_{1} f, \ldots, \varphi_{m-1} f, \varphi_{m} f=\varphi_{m-1} \varphi f=h$ any two subsequent elements satisfy $\left(\varphi_{l} f, \varphi_{l+1} f\right)=\left(\varphi_{l} f, \varphi_{l} \varphi f\right)$, hence $\left(\varphi_{l} f, \varphi_{l+1} f\right) \in \operatorname{Con}(f, \varphi f) \subseteq$ $\subseteq \operatorname{Con}(e, f)$. This proves $(g, h) \in \operatorname{Con}(e, f)$, hence $Z(\pi(e, f)) \subseteq \operatorname{Con}(e, f)$. Since $\operatorname{Con}(e, f) \subseteq Z(\pi(e, f))$ holds generally, we get $\operatorname{Con}(e, f)=Z(\pi(e, f))$.

Now take arbitrary $\pi \in \Pi(I)$ greater than an atom. The induction hypothesis is that $Z(\pi(g, h))=\operatorname{Con}(g, h)$ whenever $\pi ! g, h)<\pi$. Let us suppose that $\pi(e, f)=\pi$, and denote by $\varrho$ the greatest element of $\Pi(I)$ such that $Z(\varrho) \subseteq \operatorname{Con}(e, f)$. We have to prove $\varrho=\pi$. Suppose on the contrary $\varrho<\pi$. Then there are two different blocks $J$ and $K$ of $\varrho$ which are subsets of the same block $L$ of $\pi$. Now $J$ is not a block of $\pi$, hence $e(J) \neq f(J)$. By the condition (1.1), we find an element $l \in L-J$ such that $e^{\prime} l$ ) intersects both $f(J)$ and $f(L-J)$. Take arbitrary $x \in f(J) \cap e(l)$ and $y \in f(L-J) \cap$ $\left.\cap e^{\prime} l\right)$.

We have $x \in f(i)$ and $y \in f(j)$ for some $i \in J$ and $j \in K$. The transposition $\psi=$ $=(x, y)$ maps $e$ to $e$ and $f$ to a $p$-partition $\psi f \neq f$, since $\psi f(i) \neq f(i)$. On the other hand, $\psi f(k)=f(k)$ for all $k \neq i, j$ and $\psi f(\{i, j\})=f(\{i, j\})$. Hence we get that $\pi(f, \psi f)$ is the atom of $\Pi(I)$ having just one non-trivial block $\{i, j\}$, and $(f, \psi f) \in$ $\in \operatorname{Con}(e, f)$. By the induction hypothesis we get $Z(\pi(f, \psi f))=\operatorname{Con}(f, \psi f)$, hence $Z(\pi(f, \psi f)) \subseteq \operatorname{Con}(\mathrm{e}, f)$. It follows that $i, j$ are contained in the same block of $\varrho$, contrary to our assumption. This contradiction proves $\operatorname{Con}(e, f)=Z(\pi(e, f))$ for any pair $(e, f) \in E \times E$.

Since each join-irreducible element of $\operatorname{Con}(E, G)$ is of the form $\operatorname{Con}(e, f)$ for some $(e, f) \in E \times E($ see $[8])$, we have $\operatorname{Con}(E, G) \subseteq \operatorname{Im}(Z)$.

2. Representations of Boolean lattices. In this section we present a construction of Boolean lattices as congruence lattices of permutational algebras. The construction is a known result in group theory and is a special case of more general properties of groups with a BN-pair - see [3], [10]. We give here a direct, although somewhat lengthy, proof without any use of the theory of BN-pairs. Other examples of permutational algebras with congruence lattices isomorphic to arbitrary Boolean lattices are obtained as corollaries of constructions presented in Section 4, so this section might seem superfluous. But permutational algebras constructed in this section have 
a very special position among all permutational algebras of type $A_{n}$. This fact will be another topic of the second part of the paper.

In this section it is also more convenient to have some order on the set $I$, so we assume again $I=\{0,1, \ldots, n-1\}$. By $P$ we denote a finite projective space of rank $n+1$. We consider a projective space as a collection of points and lines subjected to some - e.g. Veblen-Young's - axioms. The rank function on subspaces of $P$ is denoted by $r$.

By a maximal flag in $P$ we mean a sequence $f=\left(f_{-1}, f_{0}, \ldots, f_{n}\right)$ of subspaces of $P$ ordered linearly by inclusion and such that $r\left(f_{i}\right)=i+1$ for all $i=-1,0, \ldots, n$. Hence $f_{-1}=\emptyset$ and $f_{n}=P$. The set of all maximal flags is denoted by $E$. The group $\mathrm{Col}(P)$ of all collineations of $P$ maps maximal flags to maximal flags and acts in this way on the set $E$. The action is obviously transitive. The permutation of $E$ induced by a collineation $\varphi \in \operatorname{Col}(P)$ (the action of $\varphi$ on $E$ ) will be also denoted by $\varphi$. We denote by $G$ the group of permutations of $E$ induced by collineations of $P$.

We shall prove $\left.\operatorname{Con}(E, G) \simeq B_{i}^{\prime} I\right)$. To this end we define a mapping $\left.Z: B^{\prime} I\right) \rightarrow$ $\rightarrow \Gamma(E)$ by

$$
(e, f) \in Z(J) \text { iff } e_{i}=f_{i} \text { for all } i \in I-J .
$$

We have the following theorem.

Theorem 2A. The mapping $Z: B(I) \rightarrow \Pi(E)$ defined by (2.1) is a complete lattice embedding.

Proof. We have $(e, f) \in Z(\emptyset)$ iff $e_{i}=f_{i}$ for all $i \in I$ iff $e=f$, and $(e, f) \in Z(I)$ for all couples $(e, f) \in E \times E$. This proves that $Z$ preserves the least and the greatest elements. A straightforward verification also shows that $Z$ preserves the order relations.

It follows that $Z(J \cap K) \subseteq Z(J) \cap Z(K)$ for any two subsets $J, K$ of $I$. But if $(e, f) \in Z(J) \cap Z(K)$, then $e_{i}=f_{i}$ for all $i \in(I-J) \cup(I-K)=I-(J \cap K)$, hence $(e, f) \in Z(J \cap K)$ and $Z(J) \cap Z(K) \subseteq Z(J \cap K)$. This proves that $Z$ preserves meets.

We get also $Z(J) \vee Z(K) \subseteq Z(J \cup K)$, since $Z$ preserves order relations. Assume $(e, f) \in Z(J \cup K)$, and set $I(e, f)=\left\{i \in I: e_{i} \neq f_{i}\right\}$. Then $I(e, f) \subseteq J \cup K$. We prove $(e, f) \in Z(J) \vee Z(K)$ by induction on the set $I(e, f)$. If $I(e, f)=\{i\}$, then either $i \in J$ or $i \in K$. In both cases we get $(e, f) \in Z(J) \vee Z(K)$. If $I(e, f)$ contains at least two elements, let us take the least possible $i \in I(e, f)$ and denote by $j$ the least element of $I-I(e, f)$ greater than $i$. Then the whole interval $[i, j-1]$ is contained in $I(e, f)$. Take a chain $a_{i+1}=e_{i} \vee f_{i} \subset a_{i+2} \subset \ldots \subset a_{j}=e_{j}=f_{j}$ of subspaces of $P$ and complete it to maximal flags

$$
\begin{aligned}
& a=\left(e_{-1}, e_{0}, \ldots, e_{i}, a_{i+1}, a_{i+2}, \ldots, a_{j}, e_{j+1}, \ldots, e_{n}\right), \\
& b=\left(f_{-1}, f_{0}, \ldots, f_{i}, a_{i+1}, a_{i+2}, \ldots, a_{j}, e_{j+1}, \ldots, e_{n}\right), \\
& c=\left(f_{-1}, f_{0}, \ldots, f_{i}, a_{i+1}, a_{i+2}, \ldots, a_{j}, f_{j+1}, \ldots, f_{n}\right) .
\end{aligned}
$$

Then $I(e, a) \subseteq[i+1, j-1], I(a, b)=\{i\}, I(b, c) \subseteq I(e, f)-[i, j-1]$ and 
$I(c, f) \subseteq[i+1, j-1]$. All these sets are proper subsets of $I(e, f)$, hence $(e, a)$, $(a, b),(b, c),(c, f) \in Z(J) \vee Z(K)$, by the induction hypothesis. This proves $(e, f) \in$ $\in Z(J) \vee Z(K)$, therefore $Z(J \cup K) \subseteq Z(J) \vee Z(K)$, and $Z$ is 1 complete lattice homomosphism.

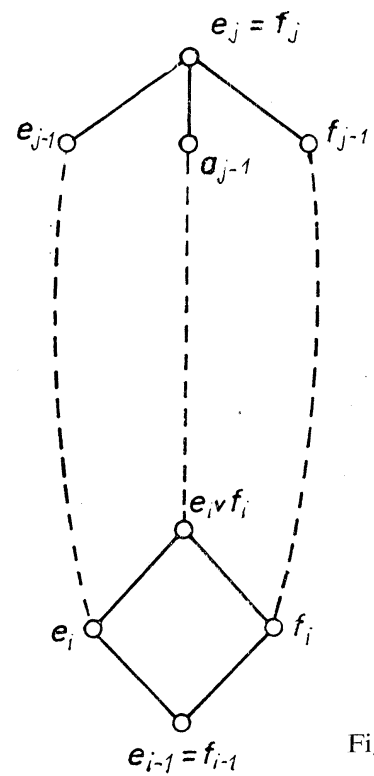

Since this homomorphism is obviously injective, the theorem is proved.

We shall need some information about the collineation group of a projective space. By a frame in a projective space $Q$ we mean a set of $k+1=r(Q)+1$ points $x_{0}, \ldots, x_{k}$ of $Q$ such that the least subspace of $Q$ containing any subset of $k$ of these points is equal to $Q$. Collineations of a projective space are fully described by the Fundamental Theorem of Projective Geometry. We use the following version.

Proposition 2.1. Let $\left\{x_{0}, \ldots, x_{k}\right\}$ and $\left\{y_{0}, \ldots, y_{k}\right\}$ be two frames in $Q$. Then there is a unique collineation $\varphi$ of $Q$ such that $\varphi\left(x_{i}\right)=y_{i}$ for all $i=0,1, \ldots, k$.

We use the Fundamental Theorem to derive some results about the group $\mathrm{Stab}_{e}$ of all eiements of $\operatorname{Col}(P)$ stabilizing a given maximal flag $e$ - the so called Borel subgroup of $\operatorname{Col}(P)$. By a base compatible with $e$ we mean a sequence $x_{0}, x_{1}, \ldots, x_{n}$ of elements of $P$ such that $x_{i} \in e_{i}-e_{i-1}$ for all $i=0,1, \ldots, n$.

Lemma 2.2. a) Let $x_{0}, \ldots, x_{n}$ and $y_{0}, \ldots, y_{n}$ be two bases compatible with $e$. Then there is an element $\varphi \in \mathrm{Stab}_{e}$ such that $\varphi\left(x_{i}\right)=y_{i}$ for all $i=0,1, \ldots, n$.

b) Let $j, k$ be two different elements of $I \cup\{n\}, x, y \in e_{j}-e_{j-1}$ and $u, v \in$ $\in e_{k}-e_{k-1}$. Then there is a collineation $\varphi \in \operatorname{Stab}_{e}$ such that $\varphi(x)=y$ and $\varphi^{\prime}(u)=v$. 
c) Let $x, y \in e_{j}-e_{j-1}$. Then there is a collineation $\varphi \in \operatorname{Stab}_{e}$ such that $\varphi / e_{j-1}$ is identity, $\varphi(x)=y$, and $\varphi(Q)=Q$ for all subspaces $Q$ of $P$ containing $e_{j}$.

Proof. 1) Since $x_{i} \in e_{i}-e_{i-1}$ for all $i=0,1, \ldots, n$, the least subspace of $P$ containing all $x_{i}$ is $P$. Hence we can complete $x_{0}, \ldots, x_{n}$ by a point $x_{n+1}$ to a frame of $P$. The same holds for $y$ 's, hence we can find a collineation $\varphi \in \operatorname{Col}(P)$ such that $\varphi\left(x_{i}\right)=y_{i}$ for all $i=0,1, \ldots, n$. Since any $e_{i}$ is spanned both by $x_{0}, \ldots, x_{i}$ and by $y_{0}, \ldots, y_{i}$, we get $\varphi\left(e_{i}\right)=e_{i}$, hence $\varphi \in \operatorname{Stab}_{e}$.

b) We can complete $x, y$ and $u, v$ to bases compatible with $e$. The rest follows from a).

c) There is a collineation $\psi: e_{j} \rightarrow e_{j}$ such that $\psi / e_{j-1}$ is the identity and $\psi(x)=y$. Then $\psi\left(e_{i}\right)=e_{i}$ for all $i \leqq j$. Now take any subspace $R$ of $P$ such that $R \cap e_{j}=\emptyset$ and $r(R)=n+1-(j+1)$. Then $P$ is spanned by the subspaces $e_{j}$ and $R$. Now take any extension $\varphi \in \operatorname{Col}(P)$ of $\psi$ satisfying $\psi / R$ is identity. (Existence of $\varphi$ can be proved either directly or as a consequence of a more general Lemma 4.1.). If $Q$ is a subspace of $P$ containing $e_{j}$, then $Q$ is spanned by $e_{j}$ and $Q \cap R$. Both the subspaces are fixed by $\varphi$, hence $\varphi(Q)=Q$. This proves also $\varphi \in \operatorname{Stab}_{e}$.

Now we are ready to prove the main result of this section.

Theorem 2B. The congruence lattice $\operatorname{Con}(E, G)$ is isomorphic to $B(I)$.

Proof. We shall prove that $\operatorname{Con}(E, G)=\operatorname{Im}(Z)$. It is easy to observe that $\operatorname{Im}(Z) \subseteq$ $\subseteq \operatorname{Con}(E, G)$. Indeed, if $(e, f) \in Z(J)$ and $\varphi \in G$, then $\left.\varphi^{\prime} e_{i}\right)=\varphi\left(f_{i}\right)$ for all $i \in I-J$. This proves $(\varphi e, \varphi f) \in Z(J)$.

It remains to prove $\operatorname{Con}(E, G) \subseteq \operatorname{Im}(Z)$. Take a couple $(e, f) \in E \times E$ and denote by $\operatorname{Con}(e, f)$ the least congruence of $(E, G)$ containing $(e, f)$. Then we have $\operatorname{Con}(e, f) \subseteq Z(I(e, f))$, since $\operatorname{Im}(Z) \subseteq \operatorname{Con}(E, G)$. Further, we denote by $J_{e, f}$ the greatest subset of $I$ such that $Z\left(J_{e, f}\right) \subseteq \operatorname{Con}(e, f)$. It exists, since $Z$ is a complete lattice embedding. We have $J_{e, f} \subseteq I(e, f)$, and we shall prove that in fact equality holds. Suppose on the contrary that there is a couple $(e, f)$ such that $J_{e, f} \subset I(e, f)$ and choose the couple $(e, f)$ in such a way that $I(e, f)$ is minimal under this condition. Fix also an element $i \in I^{\prime}(e, f)-J_{e, f}$. We shall get a contradiction by deriving further properties of $I(e, f)$.

First of all we prove the following general principle.

$$
\text { If }(g, h) \in \operatorname{Con}(e, f) \text { and } g_{i} \neq h_{i} \text {, then } I(g, h)=I(e, f) \text {. }
$$

Since $(g, h) \in \operatorname{Con}(e, f)$, we have $\operatorname{Con}(g, h) \subseteq \operatorname{Con}(e, f)$, hence $I(g, h) \subseteq I(e, f)$. If the inclusion were proper, we should have $\operatorname{Con}(g, h)=Z(I(g, h))$ by our choice of the couple $(e, f)$. But then $Z\left(J_{g, h}\right)=\operatorname{Con}(g, h) \subseteq \operatorname{Con}(e, f)$, hence $J_{g, h} \subseteq J_{e, f}$. It would imply $i \in J_{e, f}$, contrary to our assumption on $i$. This contradiction proves $I(g, h)=I(e, f)$.

Next we prove

$$
e_{i+1}=f_{i+1} \text {. }
$$


To prove it, consider the least integer $j>i$ such that $e_{j}=f_{j}$. It exists, since $e_{n}=f_{n}$. Take now a couple $(g, h) \in \operatorname{Con}(e, f)$ such that $g_{i} \neq h_{i}$ (such couples exist, e.g. $(e, f)$ ), and such that the least element of $g$ say $g_{k}$, containing $h_{i}$ has rank as large as possible under these conditions. Then $h_{i}$ contains a point $x \in g_{k}-g_{k-1}$. We have $I(g, h)=I(e, f)$ by $(2.2)$, hence $g_{j}=h_{j}$ and $k \leqq j$. If $k<j$, then $g_{k} \neq h_{k}$, and $g_{k}-g_{k-1}$ contains a point $y \in h_{l}-h_{l-1}$ for some $l>k$. Then certainly $y \notin h_{i}$. By Lemma 2.2. b), there is a collineation $\varphi \in \operatorname{Stab}_{g}$ such that $\varphi(x)=y$. Then $\varphi g=g$, hence $(g, h),(g, \varphi h) \in \operatorname{Con}(g, h)$ and also $(h, \varphi h) \in \operatorname{Con}(g, h) \subseteq$ $\subseteq \operatorname{Con}(e, f)$. We have $h_{i} \neq \varphi\left(h_{i}\right)$, since $\varphi\left(h_{i}\right)$ contains the point $y \notin h_{i}$. We have $y \in h_{l}-h_{l-1}$, hence the least element of $h$ containing $\varphi\left(h_{i}\right)$ is at least $h_{l}$ of rank

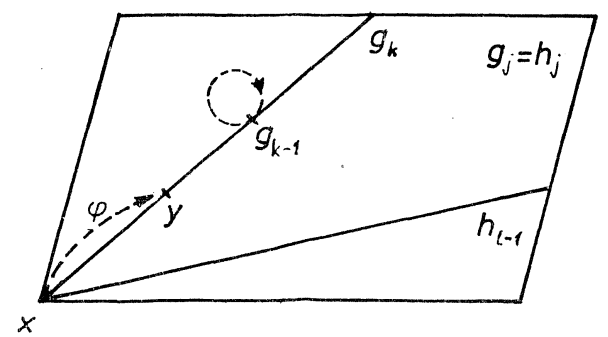

Fig. 2.2

$l+1>k+1$, contrary to our maximality assumption on the couple $(g, h)$. This proves $k=j$, hence $h_{i} \cap\left(g_{j}-g_{j-1}\right)$ is non-empty.

Now take a point $u \in h_{i} \cap\left(g_{j}-g_{j-1}\right)$ and a point $v \in\left(h_{i+1}-h_{i}\right) \cap\left(g_{j}-g_{j-1}\right)$. Such a point $v$ exists, since $h_{i} \subset h_{i+1} \subseteq g_{j}$. By Lemma 2.2. c), there is a collineation $\psi \in \mathrm{Stab}_{g}$ such that $\psi / g_{j-1}$ is the identity and $\psi(u)=v$ (see Fig. 2.3). Then $(h, \psi h) \in$ $\in \operatorname{Con}(g, h) \subseteq \operatorname{Con}(e, f)$ and $\psi\left(h_{i}\right) \neq h_{i}$, since $v=\psi(u) \notin h_{i}$. By (2.2), we have $I(h, \psi h)=I(e, f)$. But $\psi\left(h_{i+1}\right)=h_{i+1}$, since $h_{i+1} \cap g_{j-1}$ is a hyperplane of $h_{i+1}$, $u \in h_{i+1}-g_{j-1}$ and $\psi(u) \in h_{i+1}$.

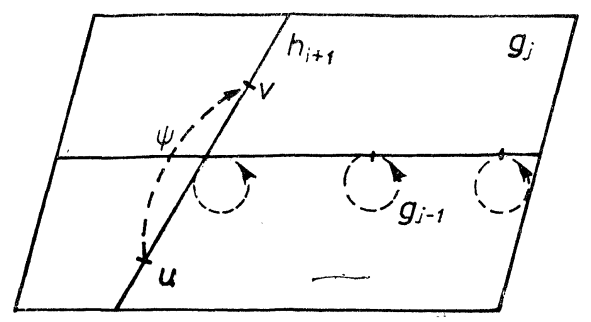

Fig. 2.3

It follows that $i+1 \notin I(h, \psi h)=I(e, f)$, hence $e_{i+1}=f_{i+1}$. The proof of $(2.3)$ is complete.

Now let us denote by $k$ the greatest element of $I \cup\{-1\}$ less than $i$ and such that $e_{k}=f_{k}$. It exists, since $e_{-1}=f_{-1}$.

$$
f_{k+1} \neq e_{i} .
$$


Suppose on the contrary that $f_{k+1} \subseteq e_{i}$. Take a point $x \in\left(f_{i}-f_{i-1}\right) \cap\left(e_{i+1}-e_{i}\right)$ " It exists since $e_{i} \neq f_{i} \subset e_{i+1}=f_{i+1}$. Next we take a point $y \in f_{i+1}-\left(f_{i} \cup e_{i}\right)$. Now apply Lemma 2.2. c) to find a collineation $\varphi \in \operatorname{Stab}_{e}$ such that $\varphi / e_{i}$ is the identity and $\varphi(x)=y$.

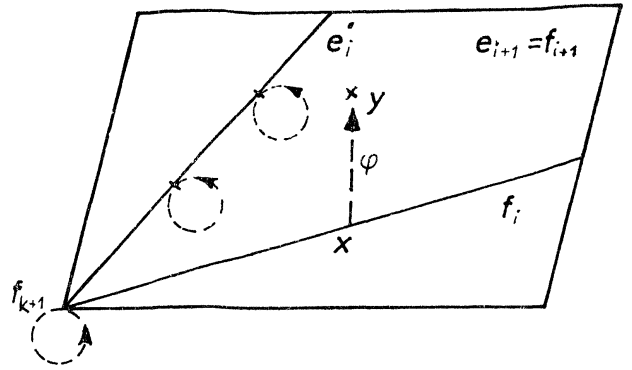

Fig. 2.4

Then $\varphi(x)=y \notin f_{i}$, hence $\left.\varphi^{\prime} f_{i}\right) \neq f_{i}$. On the other hand, $\varphi\left(f_{k+1}\right)=f_{k+1}$, since we have assumed $f_{k+1} \subseteq e_{i}$. We have $(f, \varphi f) \in \operatorname{Con}(e, f)$, hence $I(f, \varphi f)=I(e, f)$, by (2.2). This proves $k+1 \notin I(e, f)$ contrary to the fact that $k<i$ was maximal with this property. This contradiction proves $f_{k+1} \neq e_{i}$.

From the symmetry we get also

$$
e_{k+1} \nsubseteq f_{i} \text {. }
$$

Now we prove

$$
e_{i-1}=f_{i-1} \text {. }
$$

Suppose on the contrary that $e_{i-1} \neq f_{i-1}$ or, in other words, $k<i-1$. Let $w \in f_{k+1}-e_{i}$ and $z \in e_{k+1}-f_{i}$ be arbitrary points. We also find a point $x \in$ $\in\left(f_{i} \cap e_{i}\right)-f_{i-1}$. It exists, since $f_{i-1} \neq e_{i}$. Suppose that $e_{l}$ is the least element of $e$ containing $x$. Then $k<l \leqq i$. There is a point $y$ on the line joining $x$ and $z$ and different from both $x$ and $z$. Then $y \in e_{l}-e_{l-1}$. Moreover, since $z \notin f_{i}$ and $x \in f_{i}$, we have $y \notin f_{i}$. By Lemma 2.2. b), there is a collineation $\varphi \in \operatorname{Stab}_{e}$ such that $\varphi(x)=y$ and $\varphi(w)=w$.

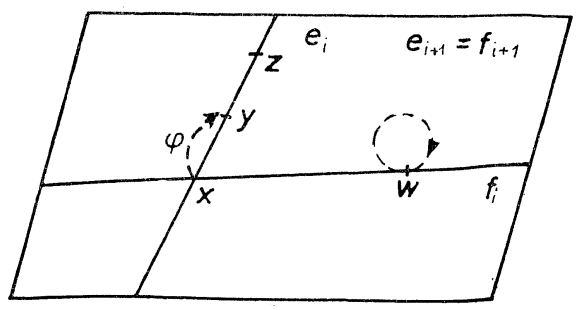

Fig. 2.5

Then $\varphi\left(f_{i}\right) \neq f_{i}$, since $y=\varphi(x) \notin f_{i}$. On the other hand, $\varphi\left(f_{k}\right)=f_{k}=e_{k}$ and $\varphi(w)=$ $=w$, therefore $\varphi\left(f_{k+1}\right)=f_{k+1}$. This proves $k+1 \notin I(f, \varphi f)$. Since $(f, \varphi f) \in \operatorname{Con}(e, f)$, we have $I(f, \varphi f)=I(e, f)$, by (2.2). But then $k+1 \notin I(e, f)$, contrary to the maxi- 
mality of $k$. This contradiction proves $k=i-1$, hence $e_{i-1}=f_{i-1}$.

$$
I(e, f)=\{i\} .
$$

Take a point $x \in f_{i}-e_{i}$ and a point $y \in e_{i+1}-\left(f_{i} \cup e_{i}\right)$. By Lemma 2.2. c), we find a collineation $\varphi \in \operatorname{Stab}_{e}$ such that $\varphi / e_{i}$ is the identity, $\varphi(x)=y$ and $\varphi(Q)=Q$ for all subspaces $Q \supseteq \boldsymbol{e}_{i+1}$.

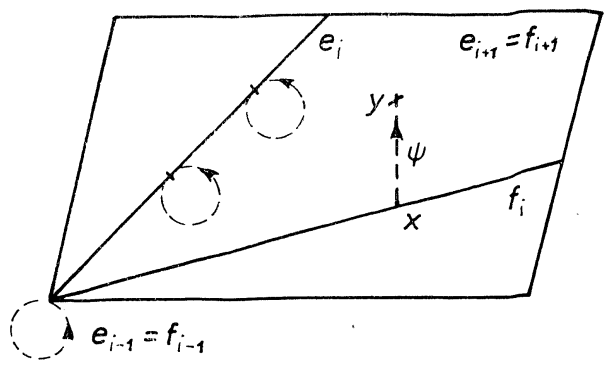

Fig. 2.6

Then $\varphi\left(f_{i}\right) \neq f_{i}$, since $y=\varphi(x) \notin f_{i}$. On the other hand, $\varphi\left(f_{k}\right)=f_{k}$ for all $k \leqq$ $\leqq i-1$, since $\varphi / e_{i-1}$ is the identity and $f_{k} \subseteq f_{i-1}=e_{i-1}$. Moreover, $\varphi\left(f_{j}\right)=f_{j}$ for all $j \geqq i+1$, since $f_{j} \supseteq f_{i+1}=e_{i+1}$. Hence $\{i\}=I(f, \varphi f)$. We have $(f, \varphi f) \in$ $\in \operatorname{Con}(e, f)$, therefore $I(f, \varphi f)=I(e, f)$, by (2.2). This completes the proof of (2.7).

$$
Z(\{i\})=\operatorname{Con}(e, f) \text {. }
$$

Take a point $x_{i+1} \in f_{i}-e_{i}$ and complete it to a base $x_{0}, \ldots, x_{n}$ compatible with $e$. If $(g, h) \in Z(\{i\})$ is another couple of mutually different maximal flags, we have $I(g, h)=\{i\}$. There is a point $y_{i+1} \in h_{i}-g_{i}$, and we complete $y_{i+1}$ to a base $y_{0}, \ldots, y_{n}$ compatible with $g$. There is a collineation $\psi$ of $P$ such that $\psi\left(x_{j}\right)=y_{j}$ for all $j=0,1, \ldots, n$.

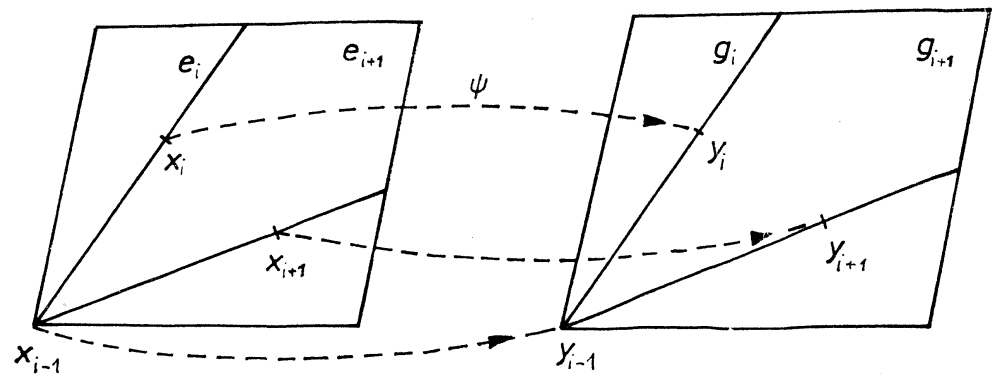

Fig. 2.7

Since each $e_{j}$ is spanned by $x_{0}, \ldots, x_{j}$ and each $g_{j}$ by $y_{0}, \ldots, y_{j}$, we get $\psi e=g$. Moreover, $f_{i}$ is spanned by $x_{0}, \ldots, x_{i-1}, x_{i+1}$ and $h_{i}$ by $y_{0}, \ldots, y_{i-1}, y_{i+1}$, hence also $\psi f=h$. This proves $(g, h) \in \operatorname{Con}(e, f)$, therefore $Z(\{i\})=\operatorname{Con}(e, f)$. 
But (2.8) contradicts our assumption on the couple $(e, f)$, hence in fact $\operatorname{Con}(e, f)=$ $=Z(I(e, f))$ for all couples $(e, f) \in E \times E$. Since all join-irreducible elements of $\operatorname{Con}(E, G)$ are of the form $\operatorname{Con}(e, f)$ for some couple $(e, f) \in E \times E$ (see [8]), we get $\operatorname{Con}(E, G) \subseteq \operatorname{Im}(Z)$.

3. Quasi-ordering lattices. In this section we define and prove some simple properties of quasi-ordering lattices. A quasi-ordering on a set $I$ is a reflexive and transitive relation on $I$. Intersection of any collection of quasi-orderings on $I$ is again ordering on $I$, hence the set of all quasi-orderings forms a complete lattice, the quasi-ordering lattice on $I$. This lattice will be denoted by $\Omega(I)$. If $\alpha, \beta \in \Omega(I)$, then the meet of $\alpha$ and $\beta$ is their intersection $\alpha \cap \beta$, while the join $\alpha \vee \beta$ is the transitive closure of $\alpha \cup \beta$. The lattice $\Omega(I)$ is atomic, the atoms being the quasi-orderings $(i>j)=\{(i, i): i \in I\} \cup\{(i, j)\}$, where $i, j \in I$ are mutually different. If $\alpha$ is a quasiordering on $I$, we say that a set $J \subseteq I$ is closed in $\alpha$ if $j \in J$ whenever $(i, j) \in \alpha$ and $i \in J$. The set of all closed sets in $\alpha$ contains together with any two subsets of $I$ also their union and intersection. Since it contains also the empty set and the whole set $I$, it forms a complete sublattice $D_{\alpha}$ of $B(I)$. Conversely, if $D$ is a complete sublattice of $B(I)$, we denote by $D(i)$ the least element of $D$ containing $i$. It exists, since $I \in D$. We define a quasi-ordering $\beta$ on $I$ by $(i, j) \in \beta$ iff $D(i) \supseteq D(j)$. A straightforward verification shows $D=D_{\beta}$. The mapping $\alpha \mapsto D_{\alpha}$ establishes a bijection between the set of all quasi-orderings on $I$ and the set $\Delta(I)$ of all complete sublattices of $B(I)$. Since $D_{\alpha \cap \beta}$ is the least complete sublattice of $\left.B_{(}^{\prime} I\right)$ containing both $D_{\alpha}$ and $D_{\beta}$, and $D_{\alpha \vee \beta}=D_{\alpha} \cap D_{\beta}$, we get the following result.

Theorem 3.1. The quasi-ordering lattice $\Omega(I)$ is dually isomorphic to the lattice $\Delta(I)$ of all complete sublattices of $\left.B_{(}^{\prime} I\right)$.

This theorem will be used in the next section. We mention also a related wellknown result.

Proposition 3.2. Let $D$ be a distributive lattice and I the set of all join-irreducible elements of $D$. Denote by $\alpha$ the ordering of $D$ restricted to $I$. Then $D \simeq D_{\alpha}$.

As a corollary we get a representation of distributive lattices as intervals in quasiordering lattices.

Corollary 3.3. Any distributive lattice is isomorphic to an interval in a quasiordering lattice.

Proof. Let $D$ be a distributive lattice, $I$ the set of join-irreducible elements of $D$ and $\alpha$ the ordering of $D$ restricted to $I$. Take an element $m \notin I$ and define two (quasi-) orderings $\bar{\alpha}, \bar{\beta}$ on $J=I \cup\{m\}$ as follows:

$$
\bar{\alpha}=\alpha \cup\{(m, m)\} \quad \text { and } \quad \bar{\beta}=\alpha \cup\{(m, i): i \in J\} .
$$

Hence $m$ is incomparable with any $i \in I$ in $\bar{\alpha}$ and greater than all $i \in I$ in $\bar{\beta}$, while $\bar{\alpha}$ and $\bar{\beta}$ restricted to $I$ coincide with $\alpha$. 
What is the interval $[\bar{\alpha}, \bar{\beta}]$ in $\Omega(J)$ ? Any quasi-ordering $\bar{\gamma} \in[\bar{\alpha}, \bar{\beta}]$ is completely described by the set $K=\{\mathrm{i} \in I:(m, i) \in \bar{\gamma}\}$. From transitivity, $K$ is closed in $\alpha$. Conversely, any closed set $K$ arises in this way. Hence the interval $[\bar{\alpha}, \bar{\beta}]$ is isomorphic to the lattice of closed sets in $\alpha$ and, by Proposition 3.2, it is isomorphic to $D$.

Finally, we describe still another representation of Boolean lattices as intervals in $\Omega(I)$.

Corollary 3.4. Take the set $I=\{0,1, \ldots, n-1\}$ in its natural order $\alpha: n-1>$ $>n-2>\ldots>1>0$. The interval $[\alpha, I \times I]$ in $\Omega(I)$ is isomorphic to the Boolean lattice on an $(n-1)$-element set.

Proof. Any quasi-ordering $\beta>\alpha$ contains a pair of equivalent elements (a pair of different elements $i, j$ is equivalent in $\beta$ iff $(i, j) \in \beta$ and $(j, i) \in \beta)$. It follows that any $\beta>\alpha$ is a join of quasi-orderings $\alpha \vee(i>i+1)$ for some $i=0,1, \ldots, n-2$. Since different joins (in $\Omega(I)$ ) of quasi-orderings $\alpha \vee(i>i+1)$ are different, the assertion is proved.

Problem. Characterize intervals in quasi-ordering lattices.

4. Representations of quasi-ordering lattices. In this section $n, N, p_{i}$ denote the same objects as in Section 1. In Theorem 4B we also assume the condition (1.1).

By $P$ we denote the projective space of rank $N$ over $G F(q)$. If necessary, we denote the lattice of all subspaces of $P$ by $\mathscr{P}$, and we also denote the rank function on the subspaces of $P$ by $r$. If $R, S$ are subspaces of $P$, then $R+S$ is the least subspace of $P$ containing $R$ and $S$.

By a $p$-partition of $P$ we mean a complete lattice embedding $e: B(I) \rightarrow \mathscr{P}$ such that $r\left(e^{\prime}(i)\right)=p_{i}$. Since $\left.r_{(}^{\prime} e^{\prime}(I)\right)=N=\sum_{I} p_{i}$, we get that $\left.e_{(}^{\prime} J\right)$ and $\left.e_{(}^{\prime} I-J\right)$ are disjoint for any subset $J$ of $I$. By $E$ we denote the set of all $p$-partitions of $P$ in this section.

Theorem 3.1 shows that the quasi-ordering lattice $\Omega(I)$ is isomorphic to $\Delta^{*}(I)$ - the lattice of all complete sublattices of $B(I)$ ordered by the opposite inclusion. To represent $\Delta^{*}(I)$ as a finite congruence lattice we define a mapping $Z: \Delta^{*}(I) \rightarrow \Pi(E)$ as follows:

$$
(e, f) \in Z(D) \text { iff } \quad e(J)=f(J) \text { for all } J \in D .
$$

Now, if $\varphi$ is a collineation of $P$ and $e$ a $p$-partition of $P$, then $\varphi_{\circ} e$ is again a $p$ partition of $P$. Hence the group $\operatorname{Col}(P)$ of all collineations of $P$ acts as a group of permutations of $E$ and this action is obviously transitive. In this section we denote by $G$ the group of permutations of $E$ induced by collineations of $P$. The action of a collineation $\varphi \in \operatorname{Col}(P)$ on the set $E$ will be also denoted by $\varphi$, which should not lead to any confusion. We want to prove that $\operatorname{Con}(E, G)$ is isomorphic to $\Delta^{*}(I)$ (and therefore to $\Omega(I)$ ). We proceed in the same way as in the two previous constructions. First we prove that the mapping $Z$ is a complete lattice embedding and then we show that $\operatorname{Im}(Z)=\operatorname{Con}(E, G)$. 
The following concept will be used throughout the whole section. Let $Q$ be a projective space and $Q=R+S$, where $R$ and $S$ are disjoint non-empty subspaces of $Q$ (a direct decomposition of $Q$ ). Suppose further that $x \notin R$ is a point of $Q$. Then the intersection $(R+x) \cap S$ contains exactly one point. This point will be called the trace of $x$ in $S$ and denoted by $\operatorname{tr}(x, S)$. If $x \in R$, then no trace of $x$ in $S$ is defined. Importance of traces follows from the fact that, if $x \notin R \cup S$, the line $\operatorname{tr}^{\prime}(x, R)+$ $+\operatorname{tr}^{\prime}(x, S)$ is the unique line through $x$ intersecting both $R$ and $S$. More generally, if $T \nsubseteq R$ is a subspace of $Q$, then the subspace $\operatorname{tr}(T, S)=(R+T) \cap S$ is the trace of $T$ in $S$. It is the set of all traces of points from $T-R$. Our notation of traces is slightly inaccurate since it does not refer to the complementary space $R$ which is not unique. However, we shall use it almost exclusively in the following context where no doubts can arise: a $p$-partition $e$ of $P$ induces a lot of direct decompositions of $P$, namely $P=e(J)+e(I-J)$ for any non-empty $J \subset I$. If $x \notin e(J)(T \notin e(J))$, then $\left.\operatorname{tr}\left(x, e^{\prime} I-J\right)\right)($ or $\operatorname{tr}(T, e(I-J)))$ is the trace of $x($ or $T)$ in $\left.e^{\prime} I-J\right)$ defined by the decomposition $P=e(J)+e(I-J)$.

As a consequence of Proposition 2.1 we get the following result.

Lemma 4.1. Let $Q=R+S$ be a direct decomposition and $x \in Q-(R \cup S)$. Suppose further that (injective) collineations $\varphi: R \rightarrow Q$ and $\psi: S \rightarrow Q$ satisfying $\operatorname{Im}(\varphi) \cap \operatorname{Im}(\psi)=\emptyset$ are given, and $y$ is any point on the line $\varphi(\operatorname{tr}(x, R))+\psi(\operatorname{tr}(x, S))$ contained neither in $\varphi(R)$ nor in $\psi(S)$. Then there is a unique collineation $\sigma: Q \rightarrow Q$ such that $\sigma(x)=y, \sigma / R=\varphi$ and $\sigma / S=\psi$.

Proof. Take a frame $\operatorname{tr}(x, R)=x_{0}, x_{1}, \ldots, x_{k}$ in $R$ and a frame $\operatorname{tr}(x, S)=y_{0}$, $y_{1}, \ldots, y_{l}$ in $S$. Then also $\varphi(\operatorname{tr}(x, R)), \varphi\left(x_{1}\right), \ldots, \varphi\left(x_{k}\right)$ and $\psi(\operatorname{tr}(x, S)), \psi\left(y_{1}\right), \ldots, \psi\left(y_{1}\right)$ are frames in $\varphi(R)$ and $\psi(S)$, respectively. A straightforward verification shows that both $x, x_{1}, \ldots, x_{k}, y_{1}, \ldots, y_{l}$ and $y, \varphi\left(x_{1}\right), \ldots, \varphi\left(x_{k}\right), \psi\left(y_{1}\right), \ldots, \psi\left(y_{l}\right)$ are frames in $Q$. By Proposition 2.1, there is a (unique) collineation $\sigma: Q \rightarrow Q$ such that $\sigma(x)=y$, $\sigma\left(x_{i}\right)=\varphi\left(x_{i}\right), i=1, \ldots, k$, and $\sigma\left(y_{j}\right)=\psi\left(y_{j}\right), j=1, \ldots, l$. It follows that $\sigma$ maps $R$ to $\varphi(R)$ and $S$ to $\psi(S)$. Moreover, it has to map the unique line through $x$ intersecting $R$ and $S$ to the unique line through $y$ intersecting $\sigma(R)=\varphi(R)$ and $\sigma(S)=\psi(S)$. Therefore $\sigma(\operatorname{tr}(x, R))=\varphi(\operatorname{tr}(x, R))$ and $\sigma(\operatorname{tr}(x, S))=\psi(\operatorname{tr}(x, S))$. Hence $\sigma$ coincides with $\varphi(\psi)$ at all points of the frame $x_{0}, \ldots, x_{k}\left(y_{0}, \ldots, y_{l}\right)$, therefore $\sigma / R=\varphi(\sigma / S=\psi$, respectively) by the uniqueness part of Proposition 2.1.

If $\tau$ is another collineation satisfying the conclusion, then $\tau^{-1} \sigma$ fixes $x$ and is the identity in both $R$ and $S$. Hence it fixes all the points of the frame $x, x_{1}, \ldots, x_{k}$, $y_{1}, \ldots, y_{l}$, and is the identity by the uniqueness part of Proposition 2.1.

Two more lemmas on traces wil be useful. The first one states a transitivity property.

Lemma 4.2. Suppose that $e$ is a p-partition of $P$ and $\emptyset \subset K \subset J \subset I$. Then for any $\left.x \notin e^{\prime} I-K\right)$ we have $\operatorname{tr}(\operatorname{tr}(x, e(J)), e(K))=\operatorname{tr}(x, e(K))$.

Proof. The assumption $\left.x \notin e_{(}^{\prime}(I-K) \supset e_{(}^{\prime} I-J\right)$ implies that the both traces 
$\operatorname{tr}(x, e(K))$ and $\operatorname{tr}^{\prime}(x, e(J))$ are defined. Moreover, it gives $\operatorname{tr}(x, e(J)) \notin e(J-K)$ (otherwise we should have $x \in e(I-J)+e(J-K)=e(I-K)$ ), hence the trace $\operatorname{tr}\left(\operatorname{tr}(x, e(J)), e^{\prime}(K)\right)$ is also defined. If $x \in e(J)$, then $\operatorname{tr}(x, e(J))=x$ and the equality is trivial. The same is true if $\left.\operatorname{tr}(x, e(J)) \in e^{\prime} K\right)$, since then $\left.\left.\operatorname{tr}^{\prime}\left(\operatorname{tr}^{\prime}\left(x, e^{\prime} J\right)\right), e^{\prime} K\right)\right)=$ $=\operatorname{tr}(x, e(J))=\operatorname{tr}(x, e(K))$. In the remaining case we have well-defined traces $\left.\operatorname{tr}\left(x, e^{\prime} I-J\right)\right)$ and $\left.\operatorname{tr}\left(\operatorname{tr}\left(x, e_{(}^{\prime} J\right)\right), e(J-K)\right)$. The points $\left.\left.\operatorname{tr}\left(x, e^{\prime} I-J\right)\right), \operatorname{tr}\left(x, e^{\prime} J\right)\right)$ and $\left.\operatorname{tr}\left(\operatorname{tr}(x, e(J)), e_{(}^{\prime} J-K\right)\right)$ are vertices of a triangle, the points $\left.x \in \operatorname{tr}\left(x, e^{\prime} J\right)\right)+$ $\left.+\operatorname{tr}\left(x, e^{\prime} I-J\right)\right)$ and $\left.\left.\operatorname{tr}\left(\operatorname{tr}\left(x, e^{\prime}(J)\right), e^{\prime} K\right)\right) \in \operatorname{tr}\left(x, e_{(}^{\prime} J\right)\right)+\operatorname{tr}\left(\operatorname{tr}\left(x, e_{(}^{\prime} J\right)\right), e^{(J-K))}$ are different from these vertices, hence we may apply the Pasch axiom.

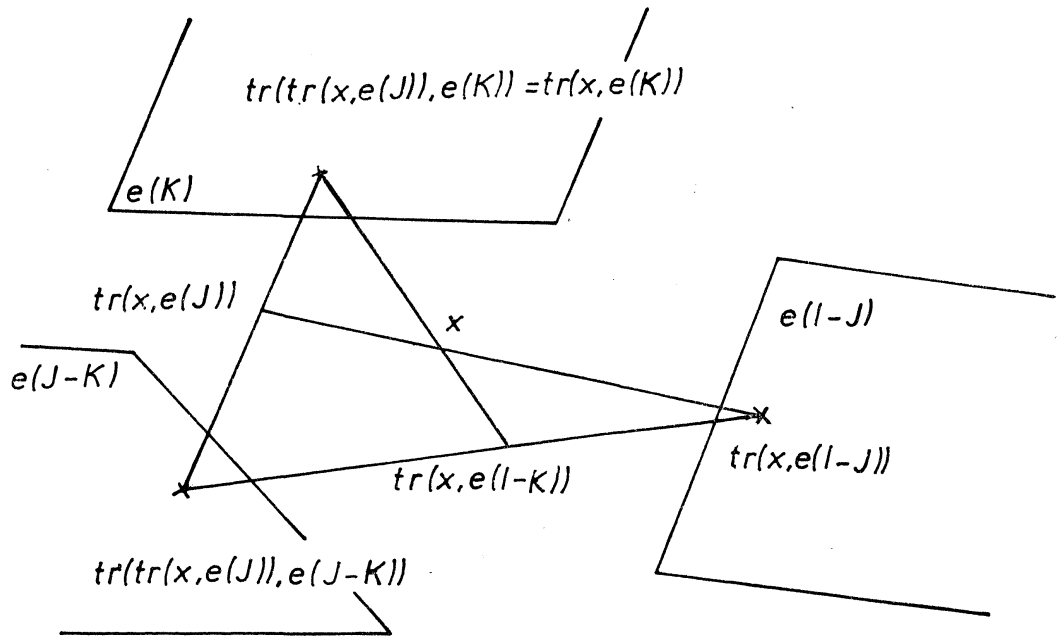

Fig. 4.1

It follows that the line $x+\operatorname{tr}\left(\operatorname{tr}\left(x, e_{(J))}^{(}, e^{\prime}(K)\right)\right.$ intersects $\operatorname{tr}(x, e(I-J))+$ $\left.+\operatorname{tr}(\operatorname{tr}(x, e(J)), \quad e(J-K)) \subseteq e^{\prime} I-K\right)$. Hence $\left.\left(e^{\prime} I-K\right)+x\right) \cap e_{(}^{\prime}(K)$ contains $\left.\operatorname{tr}\left(\operatorname{tr}(x, e(J)), e_{(}^{\prime} K\right)\right)$ and the equality is proved.

Lemma 4.3. Let $e$ be a p-partition of $P, i \in J \subseteq I$, and $x \in e(J)$ but $x \notin e(K)$ for all $K \subset J$. Suppose that $Q$ is a hyperplane in the subspace $\left.e_{(}^{(} i\right)$. Then there exists a p-partition $f$ of $P$ such that $f(i)=Q+x$ and $f(j)=e^{\prime}(j)$ for $i \neq j$ iff $Q$ does not contain $\operatorname{tr}(x, e(i))$.

Proof. The mapping $f: I \rightarrow \mathscr{P}$ can be extended to a complete lattice embedding $f: B(I) \rightarrow \mathscr{P}$ iff $(Q+x) \cap e(I-\{i\})=\emptyset$. But $\left.(Q+x) \cap e_{(}^{(} I-\{i\}\right) \subseteq(\mathrm{e}(i)+x) \cap$ $\cap e(I-\{i\})$ is non-empty iff it contains the point $\operatorname{tr}(x, e(I-\{i\}))$. Since $x \notin Q$, this is the case iff $\operatorname{tr}(x, e(i)) \in Q$.

Now we are ready to prove

Theorem 4A. The mapping $Z: \Delta^{*}(I) \rightarrow \Pi(E)$ defined by (4.1) is a complete lattice embedding. 
Proof. If $\left.D=B^{\prime} I\right)$, then $\{i\} \in D$ for any $i \in I ;(e, f) \in Z(D)$ iff $e(i)=f(i)$ for all $i \in I$ iff $e=f$. Hence $Z$ preserves the least element.

If $D=\{\emptyset, I\}$, then $(e, f) \in Z(D)$ for all couples $(e, f) \in E \times E$, hence $Z$ preserves the greatest element.

If $D \supseteq C$ and $(e, f) \in Z(D)$, then $\left.e^{\prime} J\right)=f(J)$ for all $J \in D$. But then $(e, f) \in Z(C)$ and $Z$ preserves the order relation.

From now on, $C \vee D$ and $C \cap D$ denote the join and the meet in the lattice $\Delta(I)$. Suppose that $(e, f) \in Z(C) \wedge Z(D)$. Then $e$ and $f$ coincide on all sets belonging to $C$ and $D$. Since $e$ and $f$ are lattice homomorphisms, we have $e(J)=f(J)$ for all $J$ from the least (in $\Delta(I)$ ) sublattice of $B(I)$ containing both $C$ and $D$, i.e. $C \vee D$. This proves $(e, f) \in Z(C \vee D)$. The converse inclusion $Z(C \vee D) \subseteq Z(C) \wedge Z(D)$ follows from the fact that $Z$ preserves the order relation.

For the same reason we have $Z(C) \vee Z(D) \subseteq Z(C \cap D)$. Suppose $(e, f) \in Z(C \cap D)$. We have to prove $(e, f) \in Z(C) \vee Z(D)$. By $D(e, f)$ we denote the sublattice of $B(I)$ consisting of all $J \subseteq I$ such that $\left.e_{(}^{\prime} J\right)=f(J)$. If $D(e, f)=B(I)$, then $e=f$ and $(e, f) \in$ $\in Z(C) \vee Z(D)$.

The case of $D(e, f)$ being a coatom in $\Delta(I)$ is formulated as a separate lemma.

Lemma 4.4. Suppose $D(e, f)=D_{i>j}$. Then $(e, f) \in Z(C) \vee Z(D)$.

Proof. If $D_{k>l} \supseteq C \cap D$, we define a distance of $k>l$ as follows. The assumption $D_{k>l} \supseteq C \cap D$ implies that the least element of $C \cap D$ containing $k$ contains also $l$. Hence there is a sequence $k=k_{0}, k_{1}, \ldots, k_{m}=l$ such that, for any $p=0,1, \ldots$ $\ldots, m-1$, either $k_{p+1} \in C\left(k_{p}\right)$ or $k_{p+1} \in D\left(k_{p}\right)$. The distance $d(k>1)$ is the minimum of length of all such sequences. Notice that the distance is not symmetric. Notice also that the condition $k_{p+1} \in C\left(k_{p}\right)$ is equivalent to $k_{p} \geqq_{C} k_{p+1}$ in the quasi-ordering corresponding to $C$. Hence the distance could be equivalently defined as the least length of a sequence $k=k_{0} \geqq k_{1} \geqq \ldots \geqq k_{m}=l$, where $\geqq=\geqq_{C} \cup \geqq_{D}$.

The proof proceeds by induction on $d(i>j)$. If $d(i>j)=1$, we have either $j \in C(i)$ or $j \in D(i)$. Then either $D_{i>j} \supseteq C$ or $D_{i>j} \supseteq D$. Since $D(e, f)=D_{i>j}$, we have $(e, f) \in Z(C) \vee Z(D)$ in both cases.

Suppose now that $d^{\prime}(i>j)=m>1$. The induction hypothesis is that $(a, b) \in$ $\in Z(C) \vee Z(D)$ whenever $D(a, b)=D_{k>l} \supseteq C \cap D$ and $d(k>l)<m$. Let $i=$ $=i_{0}, i_{1}, \ldots, i_{m}=j$ be a sequence defining the distance $d(i>j)$. Among all $p$-partitions $g$ satisfying $(e, g) \in Z(C) \vee Z(D)$ and $D(g, f) \supseteq D_{i>j}$ (such $p$-partitions exist, e.g. $e)$ take the one for which the intersection $f(i) \dot{\cap} g(i)$ is maximal. If $f(i) \cap g(i)$ is a proper subspace of $f(i)$, find a point $y \in f(i)-g(i)$. Since $f(j)=g(j)$, the point $z=\operatorname{tr}(y, g(i))$ is not contained in $f(i) \cap g(i)$, hence there is a hyperplane $Y$ in $g(i)$ containing $f(i) \cap g(i)$ and not containing $z$. Moreover, we denote by $w$ the trace $\operatorname{tr}(y, g(j))$. Finally, take a point $v \in g\left(i_{1}\right)$, a hyperplane $U$ of $g\left(i_{1}\right)$ not containing $v$, a point $u \in v+w, u \neq v, w$, and denote by $x$ the intersection of lines $z+u$ and $y+v$ (everything is in the plane $z+v+w$ ). 


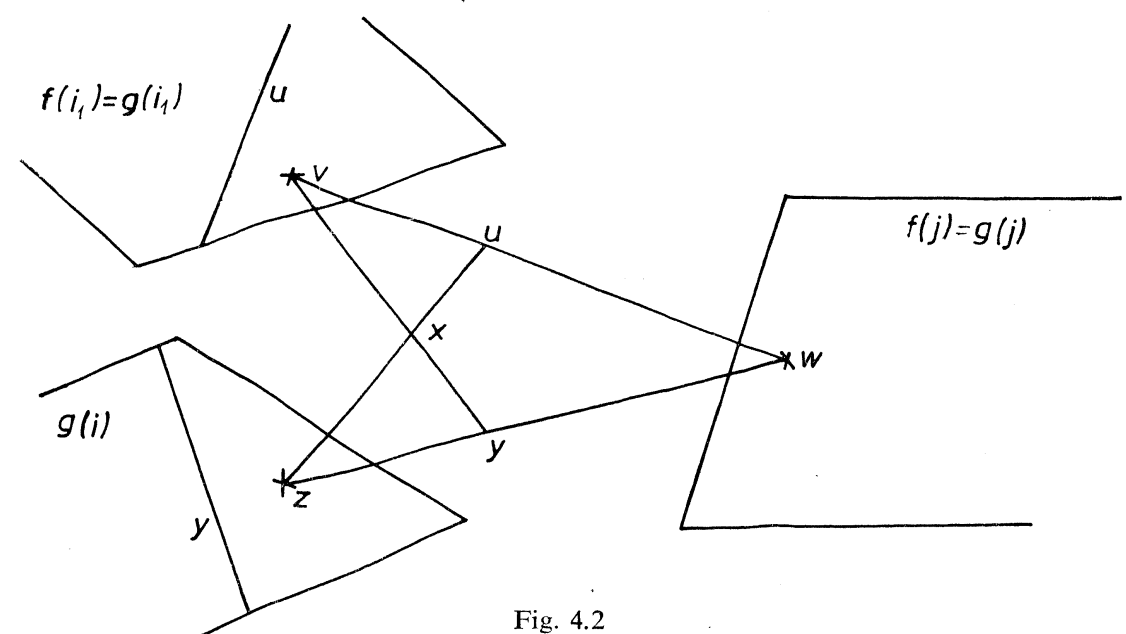

Fig. 4.2

Using Lemma 4.3, we define a p-partition $a \in E$ by $a\left(i_{1}\right)=U+u$ and $a(k)=$ $=g(k)$ for $k \neq i_{1}$. Then $D(a, g)=D_{i_{1}>j}$. But $D_{i_{1}>j} \supseteq C \cap D$ and $d\left(i_{1}>j\right)=$ $=m-1$, hence $(a, g) \in Z(C) \vee Z(D)$ by the induction hypothesis. Next, we define a $p$-partition $b$ by $b(i)=Y+x, b\left(i_{1}\right)=U+u$ and $b(k)=g(k), k \neq i, i_{1}$. Since $x \in z+u, D(a, b)=D_{i>i_{1}} \supseteq C \cap D$, and $(a, b) \in Z(C) \vee Z(D)$ since $d\left(i>i_{1}\right)=1$. Further, we define a $p$-partition $c \in E$ by $c(i)=Y+x, c\left(i_{1}\right)=g\left(i_{1}\right)$ and $c(k)=g(k)$, $k \neq i, i_{1}$. Then $D(b, c)=D_{i_{1}>j} \supseteq C \cap D$, hence $(b, c) \in Z(C) \vee Z(D)$, again by the induction hypothesis. Finally, we define a $p$-partition $d \in E$ by $d(i)=Y+y$, $d\left(i_{1}\right)=g\left(i_{1}\right)$ and $d(k)=g(k)$ for $k \neq i, i_{1}$. Since $y \in x+v$, we have $D(c, d)=$ $=D_{i>i_{1}}$. It follows, again by the induction hypothesis, that $(c, d) \in Z(C) \vee Z(D)$.

We conclude that $(g, d) \in Z(C) \vee Z(D)$, hence also $(e, d) \in Z(C) \vee Z(D)$. Moreover, $D(f, d) \supseteq D_{i>j}$ and $f(i) \cap d_{(i)} \supset f(i) \cap g(i)$, since $f(i) \cap d(i)$ contains both $f(i) \cap g(i)$ and the point $y \in f(i)-g(i)$. This contradicts our choice of $g$, hence $f(i)=g(i)$, therefore $f=g$ and $(e, f) \in Z(C) \vee Z(D)$.

The proof of $Z(C \cap D) \subseteq Z(C) \vee Z(D)$ continues by decreasing (in $\Delta(I)$ ) induction on $D(e, f)$. We have already verified that $(e, f) \in Z(C) \vee Z(D)$ if $D(e, f)$ is a coatom or the greatest element in $\Delta(I)$. The induction hypothesis is that $(g, h) \in$ $\in Z(C) \vee Z(D)$ whenever $(g, h) \in Z(C \cap D)$ and $D(g, h) \supset D(e, f)$.

Suppose now that $D(e, f)$ is less than a coatom in $\Delta(I)$. There is $i \in I$ such that $\left.\boldsymbol{e}_{(}^{(} i\right) \neq f(i)$, and denote by $J$ the least element of $D(e, f)$ containing $i$. Hence $\left.e_{(}^{\prime} K\right) \neq$ $\neq f(K)$ for all non-empty $K \subset J$ containing $i$.

First we prove that there is $g \in E$ with the properties $(e, g) \in Z(C) \vee Z(D)$, $D(g, f) \supseteq D(e, f)$ and $g(i) \cap f(J-\{i\})=\emptyset$. To this end, choose an element $g \in E$ satisfying the first and the second conditions (such elements exist, e.g. $g=e$ ), and such that $r(g(i) \cap f(J-\{i\}))$ is minimal under these conditions. Suppose on the 
contrary $g(i) \cap f(J-\{i\}) \neq \emptyset$. Then $\left.g(J-\{i\}) \supset \operatorname{tr}\left(f(J-\{i\}), \quad g^{\prime} J-\{i\}\right)\right)$, hence there is $y \in g(J-\{i\})-\operatorname{tr}(f(J-\{i\}), g(J-\{i\}))$. We take an arbitrary $x \in g(i) \cap f(J-\{i\})$ and a point $z \in x+y \quad z \neq x, y$. Since $x \in g(i)$ and $y \in$ $\in g(J-\{i\})$, we get $\operatorname{tr}(z, g(i))=x$. Now take a hyperplane $X$ in $g(i)$ not containing $x$, and use Lemma 4.3 to define a $p$-partition $h$ by $h(i)=X+z, h(j)=g(j)$ for $j \neq i$. We have $h(i) \cap f(J-\{i\}) \subset g(i) \cap f(J-\{i\})$, since $y \notin \operatorname{tr}(f(J-\{i\}), g(J-\{i\}))$. Hence $r(h(i) \cap f(J-\{i\}))<r(g(i) \cap f(J-\{i\}))$. Since $g(j)=h(j)$ for all $j \neq i$ and $z \in x+y$, we get that the least element of $D(g, h)$ containing $i$ is $J$. This proves $D(g, h) \supseteq D(e, f)$ and, because $D(g, f) \supseteq D(e, f)$, also $D(h, f) \supseteq D(e, f)$. It remains to prove that $(e, h) \in Z(C) \vee Z(D)$ and to this end $(g, h) \in Z(C) \vee Z(D)$ is sufficient.

If $D(g, h) \neq D(e, f)$, we get $(g, h) \in Z(C) \vee Z(D)$ by the induction hypothesis. If $D(g, h)=D(e, f)$, we take na element $j \in J-\{i\}$. Then $D_{i>j} \supseteq D(e, f)$, since the least element of $D(e, f)$ containing $i$ is $J$. Now consider the point $u=\operatorname{tr}(z, g\{i, j\})$ and recall that $X$ is a hyperplane in $g(i)$ not containing $\operatorname{tr}(z, g(i))$. By further application of Lemma 4.3 we construct a $p$-partition $a \in E$ satisfying $a(i)=X+u$ and $a(k)=g(k)$ for $k \neq i$. Then $\left.D^{\prime}(g, a)=D_{i>j} \supset D_{(}^{\prime} e, f\right)$, and $(g, a) \in Z(C) \vee Z(D)$ by the induction hypothesis. Moreover, $a(k)=h(k)$ for $k \neq i$ and $z \in u+$ $+\operatorname{tr}(z, h(J-\{i, j\}))$. It follows that the least element of $D(a, h)$ containing $i$ is $J-\{j\}$, hence $\left.D(a, h) \supset D^{\prime} e, f\right)$. By the induction hypothesis we get $(a, h) \in Z(C) \vee$ $\vee Z(D)$, hence also $(g, h) \in Z(C) \vee Z(D)$.

We have $(e, h) \in Z(C) \vee Z(D), \quad D(h, f) \supseteq D(e, f)$ and $r(h(i) \cap f(J-\{i\}))<$ $<r^{\prime}(g(i) \cap f(J-\{i\}))$, hence our choice of $g$ is contradicted by the $p$-partition $h$. This contradiction proves that in fact $g(i) \cap f(J-\{i\})=\emptyset$.

Now let us take a $p$-partition $b \in E$ satisfying $(b, f) \in Z(C) \vee Z(D), g(i) \cap$ $\cap b(J-\{i\})=\emptyset$ and $D(g, b) \supseteq D_{(e, f)}^{\prime}$ (such $p$-partitions exist, e.g. $f$ ), and such that $r(g(i) \cap b(i))$ is as large as possible under these conditions. Suppose that $g(i) \cap$ $\cap b(i) \subset g(i)$. Then we can find a point $v \in g(i)-b(i)$ and denote by $w$ the trace $\operatorname{tr}(v, b(i)) \in b(i)$. Since $g(i) \cap b(J-\{i\})=\emptyset$, we have also $w \notin g(i)$. Now let us take a hyperplane $Y$ in $b(i)$ containing $g(i) \cap b(i)$ and not containing $w$, and define a $p$ partition $c \in E$ by $c(i)=Y+v, c(k)=b(k)$ for $k \neq i$. The least element of $D(b, c)$ containing $i$ is then a subset of $J$. This proves $D(b, c) \supseteq D(e, f)$ and, since $D(g, b) \supseteq$

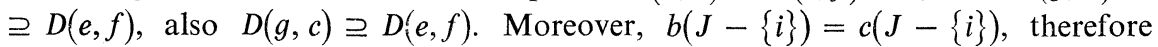
$g(i) \cap c(J-\{i\})=\emptyset$. Since $Y \supseteq g(i) \cap b(i)$ and $v \notin g(i) \cap b(i)$, we get $r(g(i) \cap$ $\cap c(i))>r(g(i) \cap b(i))$. It remains to prove $(c, f) \in Z(C) \vee Z(D)$, and to this end $(b, c) \in Z(C) \vee Z(D)$ is sufficient.

If $D(b, c) \supset D(e, f)$, then $(b, c) \in Z(C) \vee Z(D)$ by the induction hypothesis. If $D(b, c)=D(e, f)$, then $J$ is the least element of $D(b, c)$ containing $i$. This proves that all the traces $\operatorname{tr}(v, b(K)), K \subseteq J$, are defined. Now take an element $j \in J-\{i\}$ and consider the point $t=\operatorname{tr}(v, b(\{i, j\}))$. We use once more Lemma 4.3 to define a $p$-partition $d \in E$ by $d(i)=Y+t, d(k)=b(k)$ for $k \neq i$. Then $D(b, d)=D_{i>j} \supset$ $\supset D(e, f)$ and $(b, d) \in Z(C) \vee Z(D)$ by the induction hypothesis. Moreover, since $v \in t+\operatorname{tr}^{\prime}(v, c(J-\{i, j\}))$, we get that the least element of $D(c, d)$ containing $i$ is 
$J-\{j\}$, hence also $(c, d) \in Z(C) \vee Z(D)$ by the induction hypothesis. But then $(b, c) \in Z(C) \vee Z(D)$.

Our choice of $b$ is contradicted by the $p$-partition $c$, and this contradiction proves that in fact $g(i)=b(i)$.

This implies $\{i\} \in D(g, b) \supseteq D(e, f)$, hence the inclusion is in fact proper, and $(g, b) \in Z(C) \vee Z(D)$ by the induction hypothesis. Summarizing our results, we obtain $(e, g),(g, b),(b, f) \in Z(C) \vee Z(D)$. It follows that $(e, f) \in Z(C) \vee Z(D)$ which completes our proof of $Z(C \cap D)=Z(C) \vee Z(D)$.

It remains to prove that the mapping $Z$ is injective. So assume $C \subset D, C, D \in \Delta(I)$. Then there is $i \in I$ such that $D(i) \subset C(i)$. Take an arbitrary $p$-partition $e \in E$, a point $x \in e(C(i))$ such that $\left.x \notin e^{\prime} K\right)$ for all $K \subset C(i)$, and a hyperplane $Z$ in $e(i)$ not containing the trace $\operatorname{tr}\left(x, e^{\prime}(i)\right)$. Using Lemma 4.3, we define a p-partition $f$ by $f(i)=$ $=Z+x, f(j)=e(j)$ for $j \neq i$. Then the least element of $D(e, f)$ containing $i$ is $C(i)$, therefore $D(e, f) \supseteq C$ but $D(e, f) \nsupseteq D$. This proves $(e, f) \in Z(C)-Z(D)$ and the homomorphism $Z$ is a complete embedding.

Next we prove some properties of $\mathrm{Stab}_{e}$ - the group of all collineations of $P$ preserving $e$. This group is not transitive on $P$ and our first task is to describe orbits of $\mathrm{Stab}_{e}$ on $P$.

By the type of a point $x \in P$ we mean the least subset $J \subseteq I$ such that $\left.x \in e^{(} J\right)$. The type of $x$ will be denoted by $\operatorname{typ}_{e}(x)$. If $J=\operatorname{typ}_{e}(x)$, we also say that $x$ is in a general position in $e(J)$, or $x$ is a general point of $\left.e^{\prime} J\right)$. Since $\left.e_{(}^{\prime} I\right)=P$, the type of any point $x \in P$ is defined. Notice also that $\left.\operatorname{tr}\left(x, e^{\prime} I-K\right)\right)$ is defined iff $K \neq \operatorname{typ}_{e}(\dot{x})$, and, if $x$ is in a general position in $e(J)$, then $\left.\operatorname{tr}^{\prime} x, e^{\prime}(K)\right)$ is general point of $e^{\prime}(K)$ for all non-empty $K \subseteq J$.

Lemma 4.5. Two points $x, y \in P$ are in the same orbit of $\operatorname{Stab}_{e}$ iff typ $\tan _{e}(x)=\operatorname{typ}_{e}(y)$.

Moreover, suppose $\operatorname{typ}_{e}(x)=J,|J| \geqq 2$ and $K \subset J$ is non-empty. Suppose further that $\varphi, \psi \in \operatorname{Stab}_{e}$ satisfy $\left.\left.\varphi^{\prime} \operatorname{tr}^{\prime}\left(x, e^{\prime}(K)\right)\right)=\operatorname{tr}\left(y, e^{\prime} K\right)\right)$ and $\left.\psi\left(\operatorname{tr}\left(x, e^{\prime} J-K\right)\right)\right)=$ $\left.=\operatorname{tr}\left(y, e^{\prime} J-K\right)\right)$. Then there is a collineation $\tau \in \operatorname{Stab}_{e}$ such that $\tau(x)=y$, $\tau \mid e^{\prime}(K)=\varphi / e(K)$ and $\tau / e(J-K)=\psi \mid e(J-K)$.

Proof. Obviously, if $\tau(x)=y$ for some $\tau \in \operatorname{Stab}_{e}$, then $\operatorname{typ}_{e}(x)=\operatorname{typ}_{e}(y)$. The converse implication will be proved by induction on $\operatorname{typ}_{e}(x)$. Simultaneously we prove the second assertion.

If $\operatorname{typ}_{e}(x)=\{i\}$, then there is a collineation $\left.\varrho: e^{\prime} i\right) \rightarrow e^{\prime}(i)$ sending $x$ to $y$ and it can be easily extended to a collineation $\tau \in \operatorname{Stab}_{e}$. Suppose now $\left|\operatorname{typ}_{e}(x)\right| \geqq 2$ and the first assertion is true for all $u, v \in P$ such that $\left.\operatorname{typ}_{e}{ }^{\prime} u\right)=\operatorname{typ}_{e}(v) \subset \operatorname{typ}_{e}(x)$. We have $K$, $J-K \subset J$. Take the collineations $\varphi, \psi \in \mathrm{Stab}_{e}$. They exist by the induction hypothesis. By Lemma 4.1, there is a collineation $\left.\sigma: e_{(}^{\prime} J\right) \rightarrow e^{\prime}(J)$ such that $\sigma(x)=y$, $\left.\sigma / e_{i}^{\prime} K\right)=\varphi\left(e_{(}^{\prime} K\right)$ and $\left.\sigma / e(J-K)=\psi / e_{1}^{\prime} J-K\right)$. Then $\sigma(e(j))=e(j)$ for all $j \in J$, hence $\sigma$ can be extended to a collineation $\tau \in \operatorname{Stab}_{e}$.

Now we investigate orbits of $\mathrm{Stab}_{e}$ on the set of lines of $P$. First of all, we extend 
our definition of types to lines: the type of a line $l$ is the least set $J \subseteq I$ such that $l \subseteq e_{(}^{(J)}$. By the character of a line $l$ we mean the set $\operatorname{char}_{e}(l)=\left\{\operatorname{typ}_{e}(x): x \in l\right\}$ of types of points $x \in l$. A point $x \in l$ is a general point of $l$ if $\operatorname{typ}_{e}(x)=\operatorname{typ}_{e}(l)$, otherwise it is a special point of $l$. How many special points can a line $l$ have?

Lemma 4.6. A line $l$ of $P$ has at most $\left|\operatorname{typ}_{e}(l)\right|$ special points.

Proof. We have $\operatorname{typ}_{e}(x) \cup \operatorname{typ}_{e}(y)=\operatorname{typ}_{e}(l)$ for any two different points $x, y \in l$. Hence for any $i \in \operatorname{typ}_{e}(l)$ there is at most one special point $x \in l$ of a type contained in $\operatorname{typ}_{e}(l)-\{i\}$.

A line $l$ is a general line iff all points of $l$ are general, and it is an almost general line iff exactly one point of $l$ is special. We have the following characterization of general and almost general lines through their traces.

Lemma 4.7. Let $l$ be a line of $P$ and $\operatorname{typ}_{e}(l)=J$. Then

a) $l$ is a general line iff $\operatorname{tr}(l, e(j))$ is a line for all $j \in J$,

b) $l$ is an almost general line containing a special point of a type $K \subset J$ iff $\left.\operatorname{tr}\left(l, e_{(}^{\prime} K\right)\right)$ is a general line and $\operatorname{tr}(l, e(J-K))$ is a general point of $e(J-K)$.

Proof. a) Notice that $l$ contains a special point of a type $K \subset J$ iff $\operatorname{tr}(l, e(J-K))$ is a point. Indeed, $\left.\left.\left(e^{\prime} K\right)+l\right) \cap e^{\prime} J-K\right)$ is a point iff $\left.e^{\prime} K\right) \cap l$ is a point, the only point of $l$ of type $K$.

By Lemma 4.2 we get that $l$ contains a special point iff $\operatorname{tr}\left(l, e_{(j)}^{\prime}\right)$ is a point for some $j \in J$.

b) Suppose that $l$ is an almost general line. $\operatorname{Then} \operatorname{tr}(l, e(J-K))$ is a point, say $x$. If $x$ had a type $L \subset J-K$, the whole line $l$ would be contained in the subspace $\left.e^{\prime} K\right)+e(L) \subset e(J)$, a contradiction with $\operatorname{typ}_{e}(l)=J$. Hence $x$ is a general point

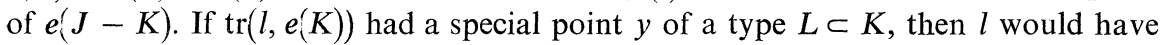
a special point $l \cap(y+x)$ of type $L \cup(J-K) \neq K$, again a contradiction.

On the other hand, if $\left.m=\operatorname{tr}\left(l, e^{\prime} K\right)\right)$ is a general line and $x=\operatorname{tr}(l, e(J-K))$ a general point, the whole line $l$ is contained in the plane $m+x$. Since $K \cap(J-K)=$ $=\emptyset$, the only non-general points contained in $m+x$ are $x$ and the points on $m$. Since $x \notin l$ (because $\operatorname{tr}(l, e(K))$ is not a point), $l$ contains exactly one special point the intersection $l \cap m-$ and the type of this special point is $K$.

As an immediate consequence of this lemma and Lemma 4.2 we get

Corollary 4.8. a) If $l$ is a general line of type $J$, then $\operatorname{tr}(l, e(K))$ is a general line for all non-empty $K \subset J$,

b) if $l$ is an almost general line containing a special point of a type $K \subset J$, then $\left.\operatorname{tr}^{\prime}\left(l, e_{(}^{\prime} L\right)\right)$ is a general line for all non-empty $L \subseteq K$ and it is an almost general line for all $L \supseteq K$.

By the "only if" part of Lemma 4.5, two lines $l, m$ of $P$ can never be in the same orbit of $\operatorname{Stab}_{c}$ on the set of lines of $P$ if $\operatorname{char}_{e}(l) \neq \operatorname{char}_{e}(m)$. However, equality of 
characters is not sufficient for $l, m$ to be in the same orbit. The following description of three special orbits is quite sufficient for our purposes.

Lemma 4.9. Suppose that $\operatorname{char}_{e}(l)=\operatorname{char}_{e}(m)$ and $l, m$ contain at most two special points $\left(\right.$ i.e. $\left.\left|\operatorname{char}_{e}(l)\right| \leqq 3\right)$. Then $l$ and $m$ are in the same orbit of $\mathrm{Stab}_{e}$ on the set of lines of $P$.

More exactly, assume that $x_{1}, x_{2}, x_{3} \in l$ and $u_{1}, u_{2}, u_{3} \in m$ are triples of different points and all the special points of $l$ and $m$ are among them. Assume further that $\operatorname{typ}_{e}\left(x_{i}\right)=\operatorname{typ}_{e}\left(u_{i}\right), i=1,2,3$. Then there is a collineation $\tau \in \operatorname{Stab}_{e}$ such that $\tau\left(x_{i}\right)=u_{i}$ for $i=1,2,3$.

If $J=\operatorname{typ}_{e}(l)$ has cardinality at least two, $K \subset J$ is nonempty and $\varphi, \psi \in \mathrm{Stab}_{e}$ satisfy $\left.\varphi\left(\operatorname{tr}\left(x_{i}, e^{\prime}(K)\right)\right)=\operatorname{tr}\left(u_{i}, e^{\prime} K\right)\right), \psi\left(\operatorname{tr}\left(x_{i}, e(J-K)\right)\right)=\operatorname{tr}^{\prime}\left(u_{i}, e(J-K)\right)$ for $i=$ $=1,2,3$, provided the traces are defined, then we can find $\tau$ satisfying in addition $\tau \mid e^{\prime}(K)=\varphi / e^{\prime}(K)$ and $\tau / e(J-K)=\psi / e(J-K)$.

Proof. We proceed by induction on cardinality of $J=\operatorname{typ}_{e}(l)$. If $J=\{j\}$, then there is a collineation $\varrho: e(j) \rightarrow e^{\prime}(j)$ such that $\varrho\left(x_{i}\right)=u_{i}, i=1,2,3$, by Proposition 2.1. This collineation can be easily extended to a collineation $\tau \in \mathrm{Stab}_{e}$.

Now assume $|J| \geqq 2$. Since $l$ and $m$ contain at most two special points, one point of each of the triples $x_{1}, x_{2}, x_{3}$ and $u_{1}, u_{2}, u_{3}$, say $x_{1} \in l$ and $u_{1} \in m$, is a general point of the corresponding line. It follows that $\operatorname{typ}_{e}\left(x_{1}\right)=\operatorname{typ}_{e}\left(u_{1}\right)=J$ and all the traces $\operatorname{tr}\left(x_{1}, e^{\prime}(j)\right), \operatorname{tr}\left(u_{1}, e^{\prime}(j)\right), j \in J$, are defined. Take arbitrary frames $\left.\operatorname{tr}\left(x_{1}, e j\right)\right)$, $y_{j, 1}, \ldots, y_{j, p_{j}}$ in $e^{(}(j), j \in J$. We set $Y_{j}=\left\{y_{j, 1}, \ldots, y_{j, p_{j}}\right\}$. Then

$$
\left.\left\{\operatorname{tr}\left(x_{1}, e^{\prime} L\right)\right)\right\} \cup \bigcup_{j \in L} Y_{j} \text { is a frame in } e(L) \text { for any non-empty } L \subseteq J .
$$

If $L=\{j\}$, the assertion is true by the definition. Assume $|L| \geqq 2$ and $Z \subset$ $\subset\left\{\operatorname{tr}\left(x_{1}, e(L)\right)\right\} \cup \bigcap_{j \in L} Y_{j}$ has cardinality $r\left(e^{\prime}(L)\right)$. We distinguish two cases.

(i) $\left.\operatorname{tr}\left(x_{1}, e_{(}^{\prime} L\right)\right) \notin Z$. Then $Y_{j} \subseteq Z$ for all $j \in L$, hence $\left.e_{(}^{\prime} j\right)$ is contained in the subspace of $e(L)$ spanned by $Z$ for all $j \in L$. Therefore $e(L)$ is spanned by $Z$.

(ii) $\left.\operatorname{tr}\left(x_{1}, e_{(}^{\prime} L\right)\right) \in Z$. Then there is a unique $k \in L$ such that $Y_{k} \neq Z$ and a unique $y_{k, l} \notin Z$. Then $Y_{j} \subseteq Z$ for all $j \in L-\{k\}$, therefore $\left.e_{(}^{\prime} L-\{k\}\right)$ is contained in the subspace spanned by $Z$. Moreover, $\operatorname{tr}\left(x_{1}, e^{\prime}(L)\right) \in Z$ and $\left.\operatorname{tr}\left(x_{1}, e(k)\right) \in \operatorname{tr}\left(x_{1}, e^{(} L\right)\right)+$ $+\operatorname{tr}\left(x_{1}, e(L-\{k\})\right)$, hence also $\left.\operatorname{tr}^{\prime}\left(x_{1}, e^{\prime} k\right)\right)$ is in the subspace spanned by $Z$. This subspace therefore contains all the points of the frame $\left.\left\{\operatorname{tr}^{\prime}\left(x_{1}, e^{\prime} k\right)\right)\right\} \cup Y_{k}$ except $y_{k, l}$. This proves that also $e^{\prime} k$ ) is in the subspace spanned by $Z$, which completes the proof of (4.2).

Suppose now that a non-empty $K \subset J$ is given. Consider collineations $\varphi, \psi \in \operatorname{Stab}_{e}$ satisfying $\left.\varphi\left(\operatorname{tr}\left(x_{i}, e^{\prime}(K)\right)\right)=\operatorname{tr}\left(u_{i}, e^{\prime} K\right)\right)$ and $\left.\left.\psi\left(\operatorname{tr}\left(x_{i}, e^{\prime} J-K\right)\right)\right)=\operatorname{tr}^{\prime}\left(u_{i}, e^{\prime} J-K\right)\right)$, provided the traces are defined, for $i=1,2,3$. Such collineations exist, either by the induction hypothesis (if $\operatorname{tr}(l, e(K))$ or $\operatorname{tr}(l, e(J-K))$ are lines) or by Lemma 4.5 (if $\left.\operatorname{tr}\left(l, e^{\prime} K\right)\right)$ or $\operatorname{tr}(l, e(J-K))$ are points). Similarly as (4.2), we prove that $\left.\left\{\operatorname{tr}\left(u_{1}, e_{(}^{\prime} K\right)\right)\right\} \cup \bigcup_{k \in K} \varphi\left(Y_{k}\right)$ is a frame in $\left.e_{(}^{\prime} K\right)$ and $\left\{\operatorname{tr}\left(u_{1}, e(J-K)\right)\right\} \cup \bigcup_{k \in J-K} \psi\left(Y_{k}\right)$ is a frame in $\left.e_{(}^{\prime} J-K\right)$, and $\left\{u_{1}\right\} \cup \bigcup_{k \in K} \varphi\left(Y_{k}\right) \cup \bigcup_{k \in J-K} \psi\left(Y_{k}\right)$ is a frame in $\left.e_{(}^{\prime} J\right)$. 
By Proposition 2.1, we find a collineation $\left.\varrho: e_{1}^{\prime} J\right) \rightarrow e(J)$ such that $\varrho\left(x_{1}\right)=u_{1}$, $\varrho\left(y_{k, l}\right)=\varphi\left(y_{k, l}\right)$ if $k \in K$ and $l=1, \ldots, p_{k}$, and $\varrho\left(y_{k, l}\right)=\psi\left(y_{k, l}\right)$ if $k \in J-K$ and $l=1, \ldots, p_{k}$. The collineation $\varrho$ maps $e(K)$ to $e(K)$, since $\varphi$ does, and $e(J-K)$ to $e(J-K)$, since $\psi$ does. Moreover, it has to map the unique line through $x_{1}$ intersecting both $e^{\prime}(K)$ and $\left.e_{(}^{(} J-K\right)$ to the unique line through $u_{1}$ intersecting both $\left.e^{\prime} K\right)$ and $e^{\prime}(J-K)$. This proves $\left.\left.\varrho\left(\operatorname{tr}\left(x_{1}, e_{(}^{\prime} K\right)\right)\right)=\varphi\left(\operatorname{tr}\left(x_{1}, e_{(}^{\prime} K\right)\right)\right)=\operatorname{tr}\left(u_{1}, e(K)\right)$ and $\left.\varrho\left(\operatorname{tr}\left(x_{1}, e(J-K)\right)\right)=\psi\left(\operatorname{tr}\left(u_{1}, e(J-K)\right)\right)=\operatorname{tr}\left(u_{1}, e_{(}^{\prime} J-K\right)\right)$. Hence $\varrho$ coincides with $\varphi$ at all points of the frame $\left\{\operatorname{tr}\left(x_{1}, e(K)\right)\right\} \cup \bigcup_{k \in K} Y_{k}$, therefore $\varrho / e^{\prime}(K)=\varphi / e(K)$ by the uniqueness part of Proposition 2.1. Similarly, $\varrho / e^{\prime}(J-K)=\psi / e(J-K)$. Moreover, $\varrho$ preserves all the subspaces $e(j), j \in J$, since $\varphi$ and $\psi$ do. So we can extend $\varrho$ to a collineation $\tau \in \operatorname{Stab}_{e}$.

It remains to prove $\tau\left(x_{2}\right)=u_{2}$ and $\tau\left(x_{3}\right)=u_{3}$. Suppose that typ $e^{\prime}\left(x_{2}\right)=\operatorname{typ}_{e}\left(u_{2}\right)=$ $=L_{2}$ and $\operatorname{typ}_{e}\left(x_{3}\right)=\operatorname{typ}_{e}^{\prime}\left(u_{3}\right)=L_{3}$. Then $L_{2} \cup L_{3}=J$. We have well-defined traces $\operatorname{tr}^{\prime}\left(x_{2}, e(j)\right), \operatorname{tr}^{\prime}\left(u_{2}, e(j)\right)$ if $j \in L_{2}$, and $\operatorname{tr}\left(x_{3}, e_{(}^{\prime}(j)\right), \operatorname{tr}\left(u_{3}, e(j)\right)$ if $j \in L_{3}$. Let us define $Q_{2}$ as the least subspace of $P$ containing the traces $\operatorname{tr}\left(x_{2}, e(j)\right), j \in L_{2}$, and $Q_{3}$ as the least subspace containing $\operatorname{tr}\left(x_{3}, e(j)\right)$ for $j \in L_{3}$. We have

$$
\left.Q_{2}=\bigcap_{j \in L_{2}}\left(e_{(}^{\prime} L_{2}-\{j\}\right)+x_{2}\right), \quad Q_{3}=\bigcap_{j \in L_{3}}\left(e_{(}^{\prime} L_{3}-\{j\}\right)+x_{3} .
$$

We can easily see that $Q_{2} \subseteq \bigcap_{j \in L_{2}}\left(e^{\prime}\left(L_{2}-\{j\}\right)+x_{2}\right)$, since each $\left.e_{(}^{\prime} L_{2}-\{j\}\right)$ contains all the traces $\left.\operatorname{tr}\left(x_{2}, e^{\prime} k\right)\right), k \in L_{2}-\{j\}$, and $e\left(L_{2}-\{j\}\right)+x_{2}$ contains both $\operatorname{tr}\left(x_{2}, e\left(L_{2}-\{j\}\right)\right)$ and $x_{2}$, hence it contains also $\operatorname{tr}\left(x_{2}, e(j)\right) \in \operatorname{tr}\left(x_{2}, e^{\prime}\left(L_{2}-\{j)\right)+\right.$ $+x_{2}$. To prove the converse inclusion, let us denote $\left.\bigcap_{j \in L_{2}} e_{(}^{\prime} L_{2}-\{j\}\right)+x_{2}$ by $R_{2}$. Notice that $\left.\left(e^{\prime} L_{2}-\{j\}\right)+x_{2}\right) \cap e(j)=\left\{\operatorname{tr}\left(x_{2}, e(j)\right)\right\}$, hence $R_{2}$ intersects each $\left.e_{(}^{\prime} j\right), j \in L_{2}$, at just one point $\operatorname{tr}\left(x_{2}, e(j)\right)$. It follows that $R_{2}$ intersects $\left.e^{\prime} L_{2}-\{j\}\right)$ in a subspace of rank $r\left(R_{2}\right)-1$. By the same argument, if $k \in L_{2}-\{j\}$, then $R_{2}$ intersects $\left.e_{(}^{\prime} L_{2}-\{j, k\}\right)$ in a subspace of rank $r\left(R_{2}\right)-2$, etc. Finally, we get that $R_{2}$ intersects $\left.e_{(}^{(} j\right)$ in a subspace of $\operatorname{rank} r\left(R_{2}\right)-\left(\left|L_{2}\right|-1\right)$. Hence $r\left(R_{2}\right)=\left|L_{2}\right|=r\left(Q_{2}\right)$. This proves $R_{2}=Q_{2}$. The second equality can be proved in the same way.

From (4.3) we conclude that $x_{2} \in Q_{2}$ and $x_{3} \in Q_{3}$. Next we prove

$$
Q_{2} \cap Q_{3}=\emptyset \text {. }
$$

We have $\operatorname{tr}\left(x_{2}, e(j)\right) \neq \operatorname{tr}\left(x_{3}, e(j)\right)$ for all $j \in L_{2} \cap L_{3}$, otherwise $l$ would contain a special point of a type contained in $J-\{j\}$, i.e. of a type different from both $L_{2}$

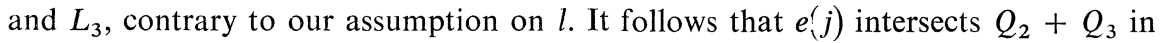
at least a line, if $j \in L_{2} \cap L_{3}$. If $j \in J-\left(L_{2} \cap L_{3}\right)$, then $e(j)$ intersects either $Q_{2}$ or $Q_{3}$ at a point. Then we get that $e(J)=Q_{2}+Q_{3}+\sum_{j \in J} e(j)$ has rank at most $\left|L_{2}\right|+\left|L_{3}\right|-r\left(Q_{2} \cap Q_{3}\right)+\sum_{j \in J} p_{j}-\left|L_{2}-L_{3}\right|-\left|L_{3}-L_{2}\right|-2\left|L_{2} \cap L_{3}\right|=$ $\left.=\sum_{j \in J} p_{j}-r_{(}^{\prime} Q_{2} \cap Q_{3}\right)$. This proves $r\left(Q_{2} \cap Q_{3}\right)=0$. 
Now we want to prove that $u_{2} \in \tau\left(Q_{2}\right)$. If $L_{2} \subseteq K$, then $\left.\operatorname{tr}^{\prime}\left(x_{2}, e^{\prime} K\right)\right)=x_{2}$ and $\tau\left(x_{2}\right)=\varphi\left(x_{2}\right)=u_{2}$, by the assumption. The same is true if $L_{2} \subseteq J-K$. If $L_{2}$ is contained neither in $K$ nor in $J-K$, we have $\left.x_{2} \in \operatorname{tr}\left(x_{2}, e K\right)\right)+\operatorname{tr}\left(x_{2}, e(J-K)\right)$, hence $\left.\tau\left(x_{2}\right) \in \tau\left(\operatorname{tr}\left(x_{2}, e^{\prime} K\right)\right)\right)+\tau\left(\operatorname{tr}\left(x_{2}, e(J-K)\right)\right)=\varphi\left(\operatorname{tr}\left(x_{2}, e(K)\right)\right)+$ $+\psi\left(\operatorname{tr}\left(x_{2}, e(J-K)\right)\right)=\operatorname{tr}\left(u_{2}, e(K)\right)+\operatorname{tr}\left(u_{2}, e(J-K)\right)$. Since both $\left.\operatorname{tr}\left(x_{2}, e_{(}^{\prime} K\right)\right)$ and $\operatorname{tr}\left(x_{2}, e(J-K)\right)$ are in $Q_{2}$, we get $u_{2} \in \tau\left(Q_{2}\right)$. Similarly we prove $u_{3} \in \tau\left(Q_{3}\right)$.

Next we consider the direct sum $Q_{2}+Q_{3}$. Since $x_{2} \in Q_{2}$ and $x_{3} \in Q_{3}, l$ is the only line through $x_{1}$ intersecting both $Q_{2}$ and $Q_{3}$. Hence $\tau(l)$ is the only line through $u_{1}=\tau\left(x_{1}\right)$ intersecting both $\tau\left(Q_{2}\right)$ and $\tau\left(Q_{3}\right)$. However, $u_{2} \in \tau\left(Q_{2}\right)$ and $u_{3} \in \tau\left(Q_{3}\right)$, therefore $\tau(l)=m, \tau\left(x_{2}\right)=u_{2}$ and $\tau\left(x_{3}\right)=u_{3}$. This completes the proof.

Now we prove some results about existence of general points. Here we also impose some restrictions on the field $\mathrm{GF}(\mathrm{q})$.

Lemma 4.10. Let $e$ be a $p$-partition of $P, p_{i} \geqq 2$ for all $i \in I$. Then for a non-empty $J \subseteq I, e(J)$ is the least subspace of $P$ containing one of the following sets of points:

a) all general points of $e(J)$,

b) all general points of $e(J)$ not contained in a given proper subspace $Q \subset e(J)$.

Proof. a) It suffices to find an almost general line through a given non-general point $x \in e(J)$. If $\operatorname{typ}_{e}(x)=K \subset J$, take a general point $y \in e(J)$ such that $\operatorname{tr}(y, e(k)) \neq$ $\neq \operatorname{tr}(x, e(k))$ for all $k \in K$. Such a point exists since $r(e(i))=p_{i} \geqq 2$ for all $i \in I$. Now consider the line $x+y$. Its trace $\operatorname{tr}(l, e(J-K))$ is a general point of $e(J-K)$, since it coincides with $\left.\operatorname{tr}\left(y, e_{(}^{\prime} J-K\right)\right)$. Moreover, $\left.\operatorname{tr}\left(l, e_{(}^{\prime} K\right)\right)$ is a general line, by Lemma 4.7a) and Lemma 4.2. By Lemma 4.7b), the line $l=x+y$ is almost general, hence it contains at least two general points. It proves that $x$ is in the least subspace of $P$ containing all general points of $e(J)$.

b) Now let $x \in Q$ be a general point in $\left.e_{(}^{(} J\right)$. Since $Q$ is a proper subspace of $\left.e_{1}^{\prime} J\right)$, there is $k \in J$ such that $e_{(k)}\left(k\right.$. Take a general point $\left.y \in e^{\prime} J\right)$ such that $\operatorname{tr}(y, e(j)) \neq$ $\left.\neq \operatorname{tr}\left(x, e^{\prime} j\right)\right)$ for all $j \in J$, and moreover $\operatorname{tr}^{\prime}\left(y, e^{\prime}(k)\right) \notin Q$. Consider the subspace $R$ spanned by the set of traces $\operatorname{tr}\left(y, e_{(j)}^{(j)}, j \in J . Q\right.$ does not contain $\operatorname{tr}(y, e(k))$, hence it intersects $R$ in a proper subspaces. This proves that we may find $y$ satisfying in addition $y \notin Q$. The line $x+y$ is a general line by Lemma $4.7 \mathrm{a}$ ) and is not contained in $Q$, therefore it contains at least two general points outside $Q$. It follows that all the points in a general position in $e^{\prime}(J)$ are contained in the subspace spanned by the general points of $\left.e_{(}^{\prime} J\right)$ not contained in $Q$. The rest follows from part a).

Corollary 4.11. Let $p_{i} \geqq 2$ for all $i \in I$. Then

a) there is a general line through any general point in $\left.e_{(}^{\prime} J\right), J \subseteq I$,

b) there is an almost general line $\left(\right.$ in $\left.\left.e_{(}^{\prime} J\right)\right)$ through any non-general point of $e(J), J \subseteq I$.

Finally, we state immediate consequences of Lemma 4.6.

Lemma 4.12. Let $e, f$ be two $p$-partitions of $P$ and $q \geqq 2 n$. 
a) Suppose that $l$ is a line of $P, \operatorname{char}_{e}(l)=J$ and $\operatorname{char}_{f}(l)=K$. Then $l$ contains a point $x$ in a general position in both $\left.e^{\prime} J\right)$ and $f(K)$.

b) Suppose that $f(K)$ is the least element of $\operatorname{Im}(f)$ containing $\left.e_{(}^{\prime} J\right)$. Then there is an element $x \in e(J)$ in a general position in both $\left.e_{(}^{\prime} J\right)$ and $f(K)$.

Proof. a) The line $l$ has $q+1 \geqq 2 n+1$ elements. At most $|J|$ of them are not general points of $e(J)$ and at most $|K|$ of them are not general points of $f(K)$, by two applications of Lemma 4.6. Hence at most $|J|+|K| \leqq 2 n$ points of $l$ are not in a general position in both $e(J)$ and $f(K)$.

b) Among all points in a general position in $\left.e_{(}^{(} J\right)$ let us find a point $x$ such that $\operatorname{typ}_{f}(x)$ is maximal. Since $e(J) \subseteq f(K)$, we have typ $\operatorname{pos}_{f}(x) \subseteq K$. Suppose that the last inclusion is proper. Since $f(K)$ is the least element of $\operatorname{Im}(f)$ containing $e(J)$, there exists a point $\left.z \in e^{\prime} J\right)$ not contained in $f\left(\operatorname{typ}_{f}(x)\right)$. The line $l=x+z$ then has characters $\operatorname{char}_{e}(l)=J$ (because of $x$ ) and $\operatorname{char}_{f}(l) \supset \operatorname{typ}_{f}(x)$ (because of $z$ ). By part a), there is a point $y \in x+z$ with the types $\operatorname{typ}_{e}(y)=J$ and $\operatorname{typ}_{f}(y)=\operatorname{char}_{f}(l) \supset$ $\supset \operatorname{typ}_{f}(x)$, contrary to our choice of $x$. Hence $x$ is a general point of $f(K)$.

Now we start to prove the main result of this paper.

Theorem 4B. Suppose that $q \geqq 3 n-2, p_{i} \geqq 2$ for all $i \in I$ and the vector $p=$ $=\left(p_{0}, p_{1}, \ldots, p_{n-1}\right)$ satisfies condition (1.1). Then the congruence lattice $\left.\operatorname{Con}^{\prime} E, G\right)$ is isomorphic to $\Omega(I)$.

Proof. We want to prove $\operatorname{Con}(E, G)=\operatorname{Im}(Z)$. We consider the mapping $Z$ as a dual embedding of $\Delta(I)$ to $\Pi(E)$. It is easy to see that $\operatorname{Im}(Z) \subseteq \operatorname{Con}(E, G)$. Indeed, if $(e, f) \in Z(D)$ for a complete sublattice $D \subseteq B(I)$, we have $\left.e_{(}^{\prime} J\right)=f(J)$ for all $J \in D$. Then also $\varphi e(J)=\varphi f(J)$ for any $\varphi \in G$, hence $(\varphi e, \varphi f) \in Z(D)$. This proves that $Z(D)$ is a congruence relation of $(E, G)$.

It remains to prove the converse inclusion $\operatorname{Con}(E, G) \subseteq \operatorname{Im}(Z)$. Let us denote by $\operatorname{Con}(e, f)$ the congruence relation of $(E, G)$ generated by a pair $(e, f) \in E \times E$, and recall that $D(e, f)=\{J \subseteq I: e(J)=f(J)\}$. Since $\operatorname{Im}(Z) \subseteq \operatorname{Con}(E, G)$, we have $\operatorname{Con}(e, f) \subseteq Z(D(e, f))$. Further, we denote by $C_{e, f}$ the least complete sublattice of $B(I)$ such that $Z\left(C_{e, f}\right) \subseteq \operatorname{Con}(e, f)$. It exists, since $Z$ is a complete embedding. Then we have $Z\left(C_{e, f}\right) \subseteq \operatorname{Con}(e, f) \subseteq Z(D(e, f))$, hence $C_{e, f} \supseteq D(e, f)$. We want to prove that in fact equality holds. It will imply that every congruence of $(E, G)$ is of the form $Z(D)$ for some $D \in \Delta(I)$.

Suppose on the contrary that there is a couple $(e, f) \in E \times E$ such that $C_{e, f} \neq$ $\neq D(e, f)$, and among all couples with this property choose the one for which $D(e, f)$ is maximal in $\Delta(I)$. Since $C=C_{e, f} \supset D(e, f)=D$, there is $i \in I$ such that the set $C(i) \in C$ - the least element of $C$ containing $i$ - is a proper subset of $D(i)$. Fix such an element $i \in I$ and set $J=D(i)$. Then we have $C(i) \subset J$. Since $J \in D$, we have $\left.e_{(}^{\prime} J\right)=f(J)$, and because $J$ is the least element of $D$ containing $i, e(K) \neq$ $\neq f(K)$ for all $K \subset J$ containing $i$.

We shall come to a contradiction by the following sequence of lemmas. Proofs of the lemmas can be omitted in the first reading, and the remaining text should 
provide a good idea about the structure of the proof. In fact, we use a multiparameter induction; however, in most cases we prefer to proceed by contradiction starting with the least possible counterexample.

First of all, let us mention that $\left.\left.D_{(}^{\prime} e, f\right) \neq B_{(}^{\prime} I\right)$. We start with a general principle.

Lemma 4.13. Let $(g, h) \in \operatorname{Con}(e, f)$ and let the least element of $D(g, h)$ containing $i$ be $J$. Then $D(g, h)=D(e, f)$.

Proof. Since $(g, h) \in \operatorname{Con}(e, f)$, we have $D(g, h) \supseteq D(e, f)$. If $D(g, h) \neq D(e, f)$, then $Z(D(g, h))=\operatorname{Con}(g, h)$ by our choice of the couple $(e, f)$. But then $Z(D(g, h)) \subseteq$ $\subseteq \operatorname{Con}(e, f)$, hence $C_{e, f} \subseteq D(g, h)$. However, $J$ is the least element of $D(g, h)$ containing $i$, therefore $C(i)=J$, contrary to our hypothesis $C(i) \neq D(i)$. Hence $\left.D(g, h)=D^{\prime} e, f\right)$.

The following lemma is of crucial importance.

Lemma 4.14. There is a couple $(g, h) \in \operatorname{Con}(e, f)$ such that $D(g, h)=D(e, f)$ and $h(i)$ contains a point in a general position in $g(J)$.

Proof. Take a couple $(g, h) \in \operatorname{Con}(e, f)$ such that $J$ is the least element of $D(g, h)$ containing $i$. Such a couple exists, e.g. $(e, f)$. We form a sequence of subspaces of $P$ as follows. Set $h\left(J_{0}\right)=h(i)$. If $h\left(J_{m}\right)$ is already defined and $h\left(J_{m}\right) \neq h(J)$, we define $g\left(K_{m}\right)$ as the least element of $\operatorname{Im}(g)$ containing $h\left(J_{m}\right)$, and $h\left(J_{m+1}\right)$ as the least element of $\operatorname{Im}(h)$ containing $g\left(J_{m}\right)$. Since $g(J)=h(J)$ and $J$ is the least element of $D(g, h)$ containing $i$ with this property, we apply the condition (1.1) to prove that $g\left(K_{m}\right)$ properly contains $h\left(J_{m}\right)$ and $h\left(J_{m+1}\right)$ properly contains $g\left(J_{m}\right)$ if $g\left(J_{m+1}\right) \neq g(J)$. This proves that the sequence is finite and ends with $h\left(J_{l+1}\right)=h(J)$. In this way we have constructed a sequence

$$
\begin{gathered}
h(i)=h\left(J_{0}\right) \subset g\left(K_{0}\right) \subset h\left(J_{1}\right) \subset \ldots \subset h\left(J_{1}\right) \subset g\left(K_{l}\right) \subseteq \\
\subseteq h\left(J_{l+1}\right)=h(J)=g(J) .
\end{gathered}
$$

Suppose now that our couple $(g, h)$ has the property that $r\left(g\left(K_{0}\right)\right)$ is maximal (under the conditions imposed in the first paragraph of the proof) and that $g\left(K_{0}\right) \subset$ $\subset g(J)$, or equivalently, $l \geqq 1$. Before proceeding further, we state a consequence of Lemma 4.12b):

(4.6) there is a point $x_{m}$ in a general position in both $h\left(J_{m}\right)$ and $g\left(K_{m}\right)$ for all $m=$ $=0,1, \ldots, l$.

Indeed, $g\left(K_{m}\right)$ is the least element of $\operatorname{Im}(g)$ containing $h\left(J_{m}\right)$.

Similarly we prove

(4.7) there is a point $u_{m}$ in a general position in $g\left(K_{m}-K_{m-1}\right)$ such that $\operatorname{typ}_{h}\left(u_{m}\right) \supseteq$ $\supseteq J_{m+1}-J_{m}$ for $m=1,2, \ldots, l$.

Take a general point $u_{m} \in g\left(K_{m}-K_{m-1}\right)$ such that typ $\operatorname{p}_{h}\left(u_{m}\right)$ is as large as possible, and suppose $\operatorname{typ}_{h}\left(u_{m}\right) \subset J_{m+1}-J_{m}$. We have $g\left(K_{m-1}\right) \subset h\left(J_{m}\right)$, and $h\left(J_{m+1}\right)$ is 
the least element of $\operatorname{Im}(h)$ containing $g\left(K_{m}\right)$. Therefore there is a point $z \in$ $\in g\left(K_{m}-K_{m-1}\right)$ such that $\operatorname{typ}_{h}(z)$ is not contained in $\operatorname{typ}_{h}\left(u_{m}\right)$. The line $u_{m}+z$ then contains a point $y$ in a general position in $g\left(K_{m}-K_{m-1}\right)$ (because of $\left.u_{m}\right)$ and of type $\operatorname{typ}_{h}(y) \supset \operatorname{typ}_{h}\left(u_{m}\right)$ (because of $z$ ), by Lemma $4.12 \mathrm{a}$ ). This contradicts our choice of $u_{m}$, hence $\operatorname{typ}_{h}\left(u_{m}\right) \supseteq J_{m+1}-J_{m}$.

Now we construct inductively a collineation $\varphi \in \operatorname{Stab}_{g}$. We can use Lemma 4.12b) to prove that there is a point $y_{0}$ in a general position in both $g\left(K_{0}\right)$ and $h\left(J_{1}\right)$. Since $\operatorname{typ}_{g}\left(x_{0}\right)=\operatorname{typ}_{g}\left(y_{0}\right)=K_{0}$, there is a collineation $\varphi_{0} \in \operatorname{Stab}_{g}$ such that $\varphi_{0}\left(x_{0}\right)=y_{0}$, by Lemma 4.5. Suppose that $\varphi_{m}, m<l$, is already defined. The points $\operatorname{tr}\left(x_{m}, g\left(K_{m+1}-K_{m}\right)\right)$ and $u_{m+1}$ are in a general position in $g\left(K_{m+1}-K_{m}\right)$, hence we can find a collineation $\psi_{m+1} \in \mathrm{Stab}_{g}$ such that $\psi_{m+1}\left(\operatorname{tr}\left(x_{m}, g\left(K_{m+1}-K_{m}\right)\right)\right)=$ $=u_{m+1}$. The line $\varphi_{m}\left(\operatorname{tr}\left(x_{m}, K_{m}\right)\right)+u_{m+1}$ contains a point $y_{m+1}$ in a general position in $g\left(K_{m+1}\right)$ (because typ be $\left.\left._{m+1}\right)=K_{m+1}-K_{m}\right)$ satisfying typ $\tan _{h}\left(y_{m+1}\right) \supseteq \operatorname{typ}_{h}\left(u_{m+1}\right) \supseteq$ $\supseteq J_{m+2}-J_{m+1}$ (by Lemma 4.12a)). Using Lemma 4.5, we find a collineation $\varphi_{m+1} \in \operatorname{Stab}_{g}$ such that $\varphi_{m+1} / g\left(K_{m}\right)=\varphi_{m} / g\left(K_{m}\right), \varphi_{m+1} / g\left(K_{m+1}-K_{m}\right)=$ $=\psi_{m+1} / g\left(K_{m+1}-K_{m}\right)$, and $\varphi_{m+1}\left(x_{m+1}\right)=y_{m+1}$.

Let us summarize important properties of $\varphi=\varphi_{l+1}$ :

$\varphi \in \mathrm{Stab}_{g}$, $\varphi\left(x_{m}\right)=y_{m}$ for all $m=0,1, \ldots, l$, $x_{m}$ is in a general position in $g\left(K_{m}\right)$, $\operatorname{typ}_{h}\left(y_{m}\right) \supseteq J_{m+1}-J_{m}$ for all $m=1,2, \ldots, l$, $y_{0}$ is in a general position in $h\left(J_{1}\right)$.

Consider the couple $(h, \varphi h)$. The subspace $\varphi h(i)$ contains the point $y_{0}=\varphi x_{0}$ in a general position in $h\left(J_{1}\right) \neq h(i)$. Hence $h \neq \varphi h$ and the least element of $\operatorname{Im}(h)$ containing $\varphi h(i)$ is $h\left(J_{1}\right) \supset g\left(K_{0}\right)$. So if we construct a sequence (4.5) for the couple $(h, \varphi h)$ instead of $(g, h)$, it starts with $\varphi h(i) \subset h\left(J_{1}\right) \subset \ldots$.

Which is the least element $K \in D(h, \varphi h)$ containing $i$ ? Since $\varphi h(i)$ contains $y_{0}$, it must be $K \supseteq J_{1}$. Suppose $K \supseteq J_{m}$ for some $m \in\{1,2, \ldots, l\}$. The subspace $\varphi h\left(J_{m}\right)$ contains the point $\varphi\left(x_{m}\right)=y_{m}$ and $\operatorname{typ}_{h}\left(y_{m}\right) \supseteq J_{m+1}-J_{m}$, hence $K \supseteq J_{m+1}$. This proves $K \supseteq J_{l+1}=J$, and in fact equality holds.

However, $r\left(h\left(J_{1}\right)\right)>r\left(g\left(K_{0}\right)\right)$, so our choice of $(g, h)$ is contradicted by the couple $(h, \varphi h)$. Therefore $g\left(K_{0}\right)=g(J)$, hence $h\left(J_{0}\right)=h(i)$ contains a point in a general position in $g(J)$. Now Lemma 4.13 gives $D(g, h)=D(e, f)$.

Since $(g, h) \in \operatorname{Con}(e, f)$, we have $\operatorname{Con}(g, h) \subseteq \operatorname{Con}(e, f) \neq D(e, f)=D(g, h)$. Moreover, $Z\left(C_{g, h}\right) \subseteq \operatorname{Con}(g, h) \subseteq \operatorname{Con}(e, f)$, therefore $C_{g, h} \supseteq C_{e, f}=C$. It follows that the least element of $C_{g, h}$ containing $i$ is contained in $C(i) \subset J$. Since $D(g, h)=$ $\left.=D_{(}^{\prime} e, f\right)$, we get that the least element of $D(g, h)$ containing $i$ is $J$. We conclude that the couple $(g, h)$ has all the properties imposed on $(e, f)$. So we may assume from now on that $f(i)$ contains a point in a general position in $\left.e_{(}^{\prime} J\right)$.

Lemma 4.15. There is a couple $(g, h) \in \operatorname{Con}(e, f)$ such that $h(i)$ contains a point $u$ 
in a general position in $g^{\prime}(J)$, and $\left.\left.\operatorname{tr}^{\prime} u, g\right)(J-\{i\})\right)$ is a point in a general position in both $g(J-\{i\})$ and $h(J-\{i\})$.

Proof. First of all, we prove

(4.8) $\operatorname{tr}(f(i), e(J-\{i\})$ is spanned by the traces of points in a general position in both $f(i)$ and $\left.e_{(}^{\prime} J\right)$.

To prove it, let us take a point $x$ in a general position in $f(i)$ and $e(J)$ and set $\left.w=\operatorname{tr}\left(x, e^{\prime} J-\{i\}\right)\right)$. If $\left.v \in \operatorname{tr}\left(f(i), e^{\prime} J-\{i\}\right)\right)$ is an arbitrary point different from $w$, there exists $z \in f(i)$ such that $v=\operatorname{tr}^{\prime}(z, e(J-\{i\}))$. The line $x+z$ is a general line in $f(i)$ and contains at most $|J| \leqq n$ non-general points of $e(J)$. Hence at least $2 n-1$ points on the line $v+w=\operatorname{tr}(x+z, e(J-\{i\}))$ are traces of points of $f(i)$ in a general position in $\left.e_{(}^{\prime} J\right)$.

Suppose now that $\left.e^{\prime} K\right)$ is the least element of $\operatorname{Im}(e)$ containing $f(J-\{i\})$. We prove similarly

(4.9) if $\left.f(J-\{i\}) \nsubseteq e^{\prime} i\right)$, then $\left.\operatorname{tr}\left(f(J-\{i\}), e_{(}^{\prime} J-\{i\}\right)\right)$ is generated by the traces of points in a general position in both $f(J-\{i\})$ and $\left.e^{\prime} K\right)$.

There is a point $z \in f(J-\{i\})$ in a general position in both $f(J-\{i\})$ and $e(K)$, by Lemma $4.13 \mathrm{~b})$. Any line in $f(J-\{i\})$ through $z$ contains at most $|J-\{i\}| \leqq$ $\leqq n-1$ points not in a general position in $f(J-\{i\})$ and $|K| \leqq n$ points not in a general position in $\left.e_{(}^{\prime} K\right)$. Hence it contains at least $n+1$ points in a general position in both $f(J-\{i\})$ and $\left.e_{(}^{\prime} K\right)$. The rest of the argument is the same as in the proof of (4.9).

Consider now the case $f(J-\{i\}) \notin e(i)$. Then $\left.\operatorname{tr}\left(f(i), e^{\prime} J-\{i\}\right)\right)+\operatorname{tr}_{(}^{\prime} f(J-\{i\})$, $\left.\boldsymbol{e}(J-\{i\}))=\boldsymbol{e}_{(}^{\prime} J-\{i\}\right)$, otherwise $f(J)$ would be contained in a proper subspace of $\left.e^{(} J\right)$. Take a maximal $L \subseteq K-\{i\}$ such that there exist a point $x_{L}$ in a general position in $f(i)$ and $\left.e^{\prime} J\right)$, and a point $y_{L}$ in a general position in $f(J-\{i\})$ and $\left.e_{(}^{\prime} K\right)$ satisfying $\left.\left.\operatorname{tr}\left(x_{L}, e_{i}^{\prime} l\right)\right) \neq \operatorname{tr}\left(y_{L}, e_{i}^{\prime} l\right)\right)$ for all $l \in L$. If there is $k \in K-L, k \neq i$, we can use (4.8) and (4.9) to find a point $x_{k}$ in a general position in $f(i)$ and $e(J)$, and a point $y_{k}$ in a general position in $f(J-\{i\})$ and $\left.e^{\prime} K\right)$ such that $\operatorname{tr}\left(x_{k}, e(k)\right) \neq \operatorname{tr}\left(y_{k}, e(k)\right)$ (we assume $p_{k}$ is at least two!).

If $\left.\left.\operatorname{tr}\left(x_{k}, e^{\prime} k\right)\right) \neq \operatorname{tr}\left(x_{L}, e^{\prime} k\right)\right)$, we certainly have $x_{k} \neq x_{L}$. The line $x_{L}+x_{k}$ contains at most $|J| \leqq n$ points not in a general position in $e(J)$, and at most $|L|+1 \leqq n-1$ points with at least one trace on some $\left.e^{\prime} m\right), m \in L \cup\{k\}$, equal to the corresponding trace of $y_{L}$. Hence we can find a general point (of $\left.\left.e_{(}^{(} J\right)\right) x_{M} \in x_{L}+x_{k}$ such that $\operatorname{tr}\left(x_{M}, e(m)\right) \neq \operatorname{tr}\left(y_{L}, e^{\prime}(m)\right)$ for all $m \in L \cup\{k\}$. This contradicts our choice of $L$.

In the case $\operatorname{tr}\left(x_{k}, e^{\prime}(k)\right)=\operatorname{tr}\left(x_{L}, e(k)\right)$, we have $\left.\operatorname{tr}\left(y_{k}, e_{(}^{\prime} k\right)\right) \neq \operatorname{tr}\left(y_{L}, e(k)\right)=$ $=\operatorname{tr}\left(x_{L}, e(k)\right)$. The line $y_{L}+y_{k}$ contains at most $|J|-1 \leqq n-1$ points not in a general position in $f(J-\{i\})$, at most $|K| \leqq n$ points not in a general position in $\left.e_{(}^{\prime} K\right)$, and at most $|L|+1 \leqq n-1$ points with at least one trace on $\left.e^{\prime} m\right), m \in$ $\in L \cup\{k\}$, equal to the corresponding trace of $x_{L}$. Since $q \geqq 3 n-2$, the lines in $P$ have at least $3 n-1$ points, hence there is a point $y_{M} \in y_{L}+y_{k}$ in a general position 
in both $\left.e^{\prime} K\right)$ and $f(J-\{i\})$, and such that $\left.\operatorname{tr}\left(x_{L}, e^{\prime}(m)\right) \neq \operatorname{tr}\left(y_{M}, e^{\prime} m\right)\right)$ for all $m \in L \cup\{k\}$.

In both cases our choice of $L$ is contradicted, hence we have $L=K-\{i\}$, i.e. $\left.\left.\left.\operatorname{tr}_{(}^{\prime} x_{L}, e^{\prime} k\right)\right) \neq \operatorname{tr}\left(y_{L}, e^{\prime} k\right)\right)$ for all $k \in K-\{i\}$. Using Lemmas 4.2 and 4.7 we can conclude that the line $\operatorname{tr}\left(x_{L}+y_{L}, e(J-\{i\})\right)$ is either an almost general line (if $K \neq J$ ) with a special point $\operatorname{tr}\left(y_{L}, e_{(}^{\prime} J-\{i\}\right)$ ), or a general line (if $K=J$ ). But then $x_{L}+y_{L}$ either has at most two special points and one of them is $y_{L}$ (if $K \neq J$ ), or has at most one special point (if $K=J$ ) and both $x_{L}$ and $y_{L}$ are general.

Take an arbitrary point $u \in x_{L}+y_{L}, u \neq x_{L}, y_{L}$, in a general position in $e(J)$. Since $\operatorname{typ}_{f}\left(x_{L}\right)=\{i\}$ and $\operatorname{typ}_{f}\left(y_{L}\right)=J-\{i\}$, we have also typ $f_{f}(u)=J$. By Lemma 4.9 , we cann find a collineation $\tau \in \mathrm{Stab}_{e}$ such that $\tau\left(x_{L}\right)=u$ and $\tau\left(u_{L}\right)=y_{L}$. Then $(f, \tau f) \in \operatorname{Con}(e, f)$ and we want to prove that $(f, \tau f)$ satisfies the conclusions of the lemma.

The point $u=\tau\left(x_{L}\right) \in \tau f(i)$ is in a general position in $f(J)$. Moreover, the point $y_{L}=\tau\left(y_{L}\right)$ is in a general position in $f(J-\{i\})$, hence also in $\tau f(J-\{i\})$. Finally, the line $x_{L}+y_{L}$ goes through $u$ and intersects both $f(i)$ and $f(J-\{i\})$, therefore $\operatorname{tr}(u, f(J-\{i\}))=y_{L}$.

It remains to consider the case $\left.f(J-\{i\}) \subseteq e^{\prime} i\right)$. But this is much easier. Take a point $y$ in a general position in $f(J-\{i\})$, a point $x \in f(i)$ in a general position in $\left.e_{(}^{(} J\right)$, and a point $u \in x+y$ in a general position in both $e(J)$ and $f(J)$ (Lemma $4.12 \mathrm{~b})$ ). The line $x+y$ contains at most two special points (in $e(J))$, and one of them is always $y$. By Lemma 4.9, we find a collineation $\tau \in \operatorname{Stab}_{e}$ with $\tau(x)=u$ and $\tau(y)=y$. The rest is the same as in the case $f(J-\{i\}) \neq e(i)$, and we prove in this way that the couple $(f, \tau f)$ satisfies the conclusions of the lemma.

Lemma 4.16. $J-\{i\} \in D(e, f)$.

Proof. Suppose on the contrary that $J-\{i\} \notin D(e, f)$. By Lemma 4.15, we find a couple $(g, h) \in \operatorname{Con}(e, f)$ such that $h(i)$ contains a point $u$ in a general position in $g(J)$ and $\operatorname{tr}(u, g(J-\{i\}))$ is a point in a general position in both $g(J-\{i\})$ and $h(J-\{i\})$. Moreover, let us assume that the couple $(g, h)$ has the property that $g(J-\{i\}) \cap h(J-\{i\})$ is maximal under these conditions.

Take a point $\left.v \in \operatorname{tr}^{\prime} u, g(i)\right)+\operatorname{tr}(u, g(J-\{i\}))$ in a general position in both $g(J)$ and $h(J)$ (Lemma 4.12a)). We distinguish two cases.

(i) $g(i) \cap h(J-\{i\}) \neq \emptyset$.

Then we use Lemma 4.5 to find a collineation $\tau \in \operatorname{Stab}_{g}$ such that $\tau(u)=v$ and both $\tau / g(i)$ and $\tau / g(J-\{i\})$ are identities. We have $(h, \tau h) \in \operatorname{Con}(g, h) \subseteq \operatorname{Con}(e, f)$ and $v \in \tau h(i)$ is in a general position in $h(J)$. Moreover, $v \in u+\operatorname{tr}(u, g(J-\{i\}))$, $u \in h(i)$ and $\operatorname{tr}(u, g(J-\{i\})) \in h(J-\{i\})$, hence $\operatorname{tr}(v, h(J-\{i\}))=\operatorname{tr}(u, g(J-\{i\}))$. However, this point is fixed by $\tau$, therefore $\left.\operatorname{tr}^{\prime} v, h(J-\{i\})\right)$ is in a general position in both $h(J-\{i\})$ and $\tau h(J-\{i\})$. This proves that the couple $(h, \tau h)$ has all the properties inposed on $(g, h)$ in the first paragraph. Moreover, $h(J-\{i\}) \cap$ 
$\left.\cap \tau h^{\prime} J-\{i\}\right)$ contains not only $g(J-\{i\}) \cap h(J-\{i\})$ (because $\tau$ is the identity on $g(J-\{i\}))$, but also the non-empty intersection $\left.g(i) \cap h^{\prime} J-\{i\}\right)$.

(ii) $g(i) \cap h(J-\{i\})=\emptyset$.

Then $\operatorname{tr}(h(J-\{i\}), g(J-\{i\}))=g(J-\{i\})$. Let us denote by $Q$ the intersection $h(J-\{i\}) \cap g(J-\{i\})$. Then $z=\operatorname{tr}(u, g(J-\{i\})) \in Q$ and $Q$ is a proper subspace of $g(J-\{i\})$. By Lemma 4.10b), there exist points in a general position in $g(J-\{i\})$ and not contained in $Q$. Suppose that $K$ is a maximal subset of $J-\{i\}$ such that there exists a point $y_{K} \in Q$ in a general position in $g(J-\{i\})$ and $\operatorname{tr}\left(y_{K}, g(k)\right) \neq \operatorname{tr}(z, g(k))$ for all $k \in K$. If $K$ is a proper subset of $J-\{i\}$, take some $l \in J-K, j \neq i$. There is a point $y_{1} \in g(J-\{i\})-Q$ such that $\operatorname{tr}\left(y_{1}, g(l)\right) \neq$ $\neq \operatorname{tr}\left(y_{K}, q(l)\right)$. Hence $y_{l} \neq y_{K}$. The line $y_{K}+y_{l}$ contains at most $|J-\{i\}| \leqq n-1$ points not in a general position in $g(J-\{i\})$ and at most $|K|+1 \leqq n-1$ points such that at least one of their traces on $g(k), k \in K \cup\{l\}$ is equal to the corresponding trace $\left.\operatorname{tr}^{\prime} z, g(k)\right)$. It follows that the line $y_{K}+y_{l}$ contains a point $y_{L}$ in a general position in $g(J-\{i\})$ and such that $\operatorname{tr}\left(y_{L}, g(k)\right) \neq \operatorname{tr}(z, g(k))$ for all $k \in K \cup\{l\}$. This contradicts our assumption on $K$, hence we get $K=J-\{i\}$.

The line $y_{K}+z$ is then a general line in $g(J-\{i\})$, by Lemma 4.7a). Since $\operatorname{tr}(h(J-\{i\}), g(J-\{i\}))=g(J-\{i\})$, we can find a point $w \in h(J-\{i\})$ such that $\operatorname{tr}(w, g(J-\{i\}))=y_{K}$. The line $u+w$ has at most one special point (if it intersects $g(J-\{i\}))$. Now we apply Lemma 4.9 and find a collineation $\tau \in$ Stab $_{g}$ such that $\tau(u)=v, \tau(w)=w$, and both $\tau / g(i)$ and $\tau / g(J-\{i\})$ are identities. As in the case (i) we prove that the couple $(h, \tau h)$ has all the properties imposed on $(g, h)$ in the first paragraph of the proof. Moreover, the intersection $h(J-\{i\}) \cap$ $\cap \tau h(J-\{i\})$ contains not only $g(J-\{i\}) \cap h(J-\{i\})$, but also the point $w \notin$ $\notin g(J-\{i\})$.

In both cases we get $h(J-\{i\}) \cap \tau h(J-\{i\}) \supset g(J-\{i\}) \cap h(J-\{i\})$. This contradiction proves that in fact $g(J-\{i\})=h(J-\{i\})$, therefore $J-\{i\} \in$ $\in D(g, h)-D(e, f)$ and $D(g, h) \supset D(e, f)$. Since $h(i)$ contains a point in a general position in $g(J), J$ is the least element of $D(g, h)$ containing $i$. But then $D(g, h) \supset$ $\supset D(e, f)$ contradicts Lemma 4.13 , hence our basic assumption $J-\{i\} \notin D(e, f)$ is false, and this proves Lemma 4.16.

Lemma 4.17. $\{j\} \in D(e, f)$ for all $j \in J-\{i\}$.

Proof. Take again a couple $(g, h) \in \operatorname{Con}(e, f)$ satisfying the conclusions of Lemma 4.15. Let $v \in \operatorname{tr}(u, g(i))+\operatorname{tr}(u, g(J-\{i\}))$ be in a general position in both $g(J)$ and $h(J)$ (Lemma 4.12a)). By Lemma 4.5, we find a collineation $\tau \in \mathrm{Stab}_{g}$ such that $\tau(u)=v$, and both $\tau / g(i)$ and $\tau / g(J-\{i\})$ are identities. We have $u \in h(i)$ and $v=\tau(u)$ in a general position in $h(J)$, hence $J$ is the least element of $D(h, \tau h)$ containing $i$. Moreover, $\tau h(j)=h(j)$ for all $j \in J-\{i\}$, since $\tau$ is the identity on $g(J-\{i\})=h(J-\{i\})$. It follows that $\{j\} \in D(g, h)$ for all $j \in J-\{i\}$. We have $(g, h) \in \operatorname{Con}(e, f)$, hence $D(g, h)=D(e, f)$ by Lemma 4.13 and $\{j\} \in D(e, f)$ for all $j \in J-\{i\}$. 
Lemma 4.18. $|J|=2$.

Proof. Suppose on the contrary that $|J| \geqq 3$ and take an arbitrary $j \in J-\{i\}$. The set $J-\{i, j\}$ is still non-empty. There exist couples $(g, h) \in \operatorname{Con}(e, f)$ such that $h(i)$ contains a point in a general position either in $g(J)$ or in $g(J-\{i\})$, e,g. $(e, f)$ has these properties. Take a particular couple $(g, h) \in \operatorname{Con}(e, f)$ such that $r(h(i) \cap g(J-\{j\}))$ is maximal under these conditions.

Assume that $h(i)-g(J-\{j\})$ is non-empty. Then $h(i)$ always contains a point in a general position in $g(J)$. Indeed, if $u \in h(i)$ is in a general position in $g(J-\{j\})$ and $v \in h(i)-g(J-\{j\})$, the line $u+v \subseteq h(i)$ contains a point in a general position in $g(J)$, since $j \in \operatorname{typ}_{g}(v)$. Next, we take a point $z$ in a general position in $g(J-\{i, j\})$. It follows that $z$ is also in a general position in $h(J-\{i, j\})$, because $g(k)=h(k)$ for all $k \in J-\{i\}$, by Lemma 4.17 and the fact that $(g, h) \in \operatorname{Con}(e, f)$. The line $x+z$ contains a point $y$ in a general position in both $g(J)$ and $h(J-\{j\})$, by Lemma 4.12a). Since $z \in g(J-\{i\})$, we have $\operatorname{tr}(x, g(i))=\operatorname{tr}(y, g(i))$. Now we apply Lemma 4.5 to find a collineation $\tau \in \operatorname{Stab}_{g}$ such that $\tau(x)=y$ and $\tau / g(i)$ is the identity. Then $(h, \tau h) \in \operatorname{Con}(g, h) \subseteq \operatorname{Con}(e, f)$. We have $y \in \tau h(i)$ in a general position in $h(J-\{j\})$.

It remains to prove that $r(\tau h(i) \cap h(J-\{j\}))>r(h(i) \cap g(J-\{j\}))$. Consider a point $w \in h(i) \cap g(J-\{j\})$. Then $\tau(w) \in \tau(\operatorname{tr}(w, g(i)))+\tau(\operatorname{tr}(w, g(J-\{i, j\})))=$ $\operatorname{tr}(w, g(i))+\tau(\operatorname{tr}(w, g(J-\{i, j\})))$. If $\tau w=w$, we have $\tau w \in h(i) \subseteq h(J-\{j\})$. If $\tau w \neq w$, the line $w+\tau w$ always intersects $h(J-\{i, j\})$, either at the point $\operatorname{tr}(w, g(J-\{i, j\}))($ if $\tau$ fixes the point $)$ or at a point on the line $\operatorname{tr}(w, g(J-\{i, j\}))+$ $+\tau(\operatorname{tr}(w, g(J-\{i, j\}))) \subseteq g(J-\{i, j\})=h(J-\{i, j\})$.

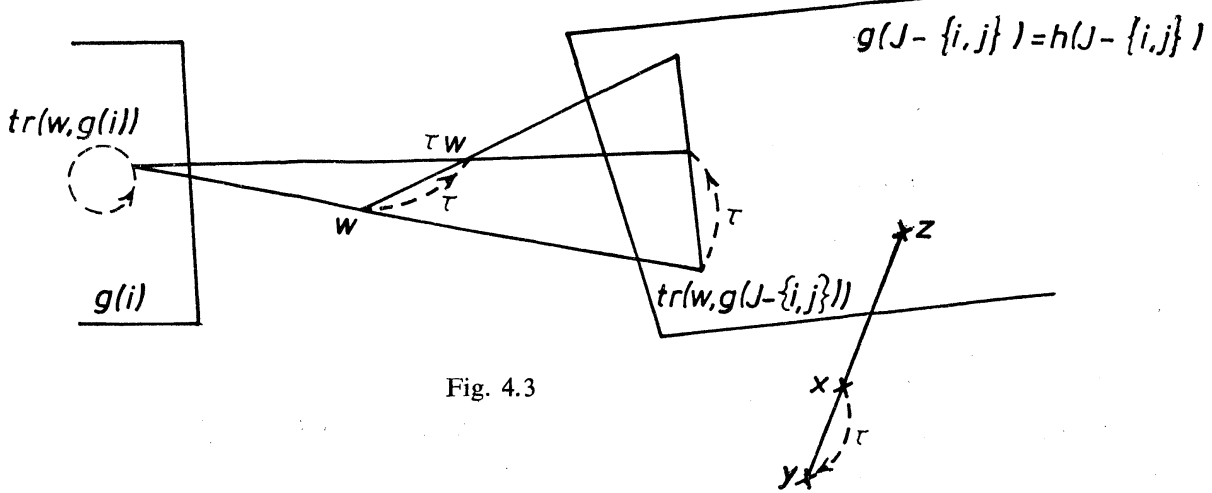

In all cases we get $\tau(w) \in h(J-\{j\})$. It follows that $\tau h(i) \cap h(J-\{j\})$ contains $\tau(h(i) \cap g(J-\{j\}))$. It contains also the point $y=\tau(x) \in x+z$. Since $x \in h(i)-$ $-g(J-\{j\})$, we get that $r(\tau h(i) \cap h(J-\{j\}))>r(h(i) \cap g(J-\{j\}))$. This contradicts our choice of the couple $(g, h)$, hence in fact $h(i) \subseteq g(J-\{j\})$.

But $g(k)=h(k)$ for any $k \in J-\{i\}$, hence $g(J-\{j\})=h(J-\{j\})$. Since $h(i)$ 
contains a point in a general position in $g(J-\{j\})$, we get that the least element of $D(g, h)$ containing $i$ is $J-\{j\}$. Since $(g, h) \in \operatorname{Con}(e, f)$, we get $D(g, h) \supset D(e, f)$. Hence $Z(D(g, h))=\operatorname{Con}(g, h) \subseteq \operatorname{Con}(e, f)$, therefore $D(g, h) \supseteq C_{e, f}=C$. It follows that the least element of $C$ containing $i$ is at least $J-\{j\}$. Since there are at least two different $j \in J-\{i\}$, we conclude that in fact $J=C(i)$, contrary to our hypothesis $C(i) \subset J$. Hence $|J|=2$.

Lemma 4.19. There is a couple $(g, h) \in \operatorname{Con}(e, f)$ such that $D(g, h)=D(e, f)$ and $r(g(i) \cap h(i))=p_{i}-1$ (i.e. $g(i) \cap h(i)$ is a hyperplane in $\left.g(i)\right)$.

Proof. Take a couple $(g, h) \in \operatorname{Con}(e, f)$ such that $D(g, h)=D(e, f)$ (such couples exist, e.g. $(e, f))$ and with $g(i) \cap h(i)$ maximal under these conditions. Then $g(i) \neq$ $\neq h(i)$, since $\{i\} \notin D(e, f)=D(g, h)$. Suppose that $r^{\prime}(g(i) \cap h(i))<p_{i}-1$. Then we can find two different points $x, y \in h(i)$ such that the line $x+y$ does not intersect $g(i) \cap h(i)$. We have $J=\{i, j\}$ and $x+y \subseteq g(J)$ is disjoint with both $g(i)$ and $\left.g^{\prime} j\right)=h(j)$, hence $x+y$ is a general line. It follows that $\operatorname{tr}(x, g(i)) \neq \operatorname{tr}(y, g(i))$ and $\operatorname{tr}^{\prime}(x, g(j)) \neq \operatorname{tr}(y, g(j))$.

Take a point $u \in \operatorname{tr}(x, g(i))+\operatorname{tr}(x, g(j)), u \neq x$ and in a general position in both $g(J)$ and $h(J)$. Then the line $u+y$ is also a general line. By Lemma 4.9 , there is a collineation $\tau \in \mathrm{Stab}_{g}$ such that $\tau(x)=u, \tau(y)=y$ and both restrictions $\tau / g(i)$ and $\tau / g(j)$ are identities. Then $(h, \tau h) \in \operatorname{Con}(g, h) \subseteq \operatorname{Con}(e, f), u=\tau(x) \in \tau h, i)$ is in a general position in $h(J)$, hence the least element of $D(h, \tau h)$ containing $i$ is again $J$. The intersection $h(i) \cap \tau h(i)$ contains not only $g(i) \cap h(i)$ (since $\tau$ is the identity on $g(i)$ ), but also the point $y \notin g(i)$. This proves $\left.h(i) \cap \tau h_{i}^{\prime} i\right) \supset g(i) \cap h(i)$. By Lemma 4.13 we get also $D(h, \tau h)=D(e, f)$. Hence our choice of the couple $(g, h)$ is contradicted by the couple $(h, \tau h)$. This contradiction proves that in fact $g(i) \cap h(i)$ is a hyperplane in $g(i)$.

We have again $Z\left(C_{g, h}\right) \subseteq \operatorname{Con}(g, h) \subseteq \operatorname{Con}(e, f)$, hence $\left.C_{g, h} \supseteq C_{e, f} \supset D e, f\right)=$ $=D(g, h)$. The least element of $D(g, h)$ containing $i$ is $J$, while the least element of $C_{g, h}$ containing $i$ is a subset of $C(i) \subset J$. Moreover, $h(i)$ contains a point in a general position in $g(J)$. It follows that the couple $(g, h)$ has all the properties we imposed on $(e, f)$, so we may change the notation and assume from now on that $\left.e_{(}^{(} i\right) \cap f(i)$ is a hyperplane in both $e(i)$ and $f(i)$.

Lemma 4.20. $D(e, f)=D_{i>j}$.

Proof. Let us recall that $J=\{i, j\}$, by Lemma 4.18, and $e(j)=f(j)$, by Lemma 4.17. First of all, we prove the following auxiliary statement.

(4.10) Let $(g, h) \in \operatorname{Con}(e, f), r(g(i) \cap h(i))=p_{i}-1$. Then for any $z \in g(J)=f(J)$, there exists a collineation $\varphi \in \operatorname{Stab}_{g}$ such that $\varphi(z)=z, \varphi h(i)$ contains a point in a general position in $h(J)$ and $h(i) \cap \varphi h(i)$ is a hyperplane in $h(i)$.

To prove it, we distinguish three cases.

(i) $z \in g(i) \cup g(j)$. 
In this case we take a point $x \in h(i)$ in a general position in $g(J)$ and a point $u \in$ $\epsilon \operatorname{tr}(x, g(i))+\operatorname{tr}(x, g(j))$ in a general position in both $g(J)$ and $h(J)$. By Lemma 4.5, there is a collineation $\varphi \in \operatorname{Stab}_{g}$ such that $\varphi(x)=u$ and both $\varphi / g(i)$ and $\varphi / g(j)$ are identities. We have $u \in \varphi h(i)$ in a general position in $h(J)$. Moreover, $h(i)$ and $\varphi h(i)$ are contained in the subspace $g(i)+x=g(i)+u$ of rank $p_{i}+1$, therefore $r(h(i) \cap$ $\cap \varphi h(i))=p_{i}-1$.

(ii) $z \notin g(i) \cup g(j) \cup h(i)$.

We can find $x \in h(i)$ in a general position in $g(J)$ and such that $\operatorname{tr}(x, g(i)) \neq \operatorname{tr}(z, g(i))$, and a point $u \in \operatorname{tr}(x, g(i))+\operatorname{tr}(x, g(j))$ in a general position in both $g(J)$ and $h(J)$. Then both the lines $x+z, u+z$ are either general (if $\operatorname{tr}(x, g(j)) \neq \operatorname{tr}(z, g(j)))$ or almost general (if the equality holds). By Lemma 4.9, there is a collineation $\varphi \in \operatorname{Stab}_{g}$ such that $\left.\varphi(x)=u, \varphi^{\prime} z\right)=z$, and both $\varphi / g(i)$ and $\varphi / g(j)$ are identities. The rest of the argument is the same as in the case (i).

(iii) $z \in h(i)-g(i)$.

In this case take a point $v \in g(i) \cap h(i)$ and a point $\left.u \in \operatorname{tr}_{(}^{\prime} z, g(i)\right)+v, u \neq v$, $\operatorname{tr}(z, g(i))$. There is a collineation $\sigma \in \operatorname{Stab}_{g}$ such that $\sigma(v)=u$ and $\sigma(\operatorname{tr}(z, g(i)))=$ $=\operatorname{tr}(z, g(i))$. By Lemma 4.5, we find a collineation $\varphi \in \operatorname{Stab}_{g}$ such that $\varphi(z)=z$, $\varphi / g(i)=\sigma / g(i)$, and $\varphi / g(j)$ is the identity. We have $u=\varphi(v) \notin h(i)$, hence $\varphi h(i) \neq$ $\neq h(i)$. Both $\varphi h(i)$ and $h(i)$ are contained in $g(i)+z$, hence $r(\varphi h(i) \cap h(i))=$ $=p_{i}-1$. This completes the proof of (4.10).

Now take an arbitrary $k \in I-J$. We choose a couple $(g, h) \in \operatorname{Con}(e, f)$ such that $r(g(i) \cap h(i))=p_{i}-1$ (such couples exist, e.g. $\left.(e, f)\right)$ and such that $g(k) \cap h(k)$ is maximal under this condition. Suppose there is a point $y \in h(k)-g(k)$. If $y \notin$ $\notin g(I-J)$, then the trace $\operatorname{tr}(y, g(J))$ is defined. Let us denote it by $z$. Take a collineation $\varphi \in \mathrm{Stab}_{g}$ constructed in (4.10) and use Lemma 4.5 to find a collineation $\tau \in \operatorname{Stab}_{g}$ such that $\tau(y)=y, \tau / g(j)=\varphi / g(j)$ and $\tau / g(I-J)$ is the deintity. If $y \in g(I-J)$, no point $z$ is defined, and we simply take an arbitrary $\varphi \in \operatorname{Stab}_{g}$ satisfying the conclusions of (4.10) (with $\varphi(z)=z$ omitted). We construct a collineation $\tau \in \operatorname{Stab}_{g}$ such that $\tau / g(J)=\varphi / g(J)$ and $\tau / g(I-J)$ is again the identity.

Then we have $(h, \tau h) \in \operatorname{Con}(e, f), r(h(i) \cap \tau h(i))=p_{i}-1$. In both cases, $h(k) \cap$ $\cap \tau h(k)$ contains not only $g(k) \cap h(k)$ but also the point $y \notin g(k)$. Hence $h(k) \cap$ $\cap \tau h(k) \supset g(k) \cap h(k)$ and our choice of $(g, h)$ is contradicted. It follows that in fact $g(k)=h(k)$.

We have $\{k\} \in D(g, h)$, and the least element of $D(g, h)$ containing $i$ is $J=\{i, j\}$. The proof of $D(g, h)=D(e, f)$ follows again from Lemma 4.13. This proves that in fact $\{k\} \in D(e, f)$ for all $k \in I-J$. Since we already know that $\{j\},\{i, j\} \in D(e, f)$, and $\{i\} \notin D^{\prime}(e, f)$, it completes the proof of $D(e, f)=D_{i>j}$.

The final contradiction is reached by the following lemma.

Lemma 4.21. $Z(D(e, f))=\operatorname{Con}(e, f)$.

Proof. We have $D(e, f)=D_{i>j}$, by the previous lemma, and $r(e(i) \cap f(i))=$ 
$=p_{i}-1$, by Lemma 4.19. Since $\operatorname{Con}(e, f) \subseteq Z(D(e, f))$, it remains to prove the converse inclusion $Z(D(e, f)) \subseteq \operatorname{Con}(e, f)$.

Take an arbitrary couple $(g, h) \in Z(D(e, f))$. We have $g(j)=h(j)$ and $g(\{i, j\})=$ $=h(\{i, j\})$. We can find a sequence $g(i)=g_{0}(i), g_{1}(i), \ldots, g_{m}(i)=h(i)$ of subspaces of $g(\{i, j\})$ such that none of them intersects $h(j)$ and the intersection of any two subsequent members is a hyperplane in both of them. We complete $g_{l}(i)$ to a $p$-partition $g_{l}$ by defining $g_{l}(k)=g(k)=h(k)$ for all $k \neq i$ and $l=0,1 \ldots, m$. Hence $g_{0}=g$ and $g_{m}=h$.

By Lemma 4.1, there are collineations $\varphi_{l}: P \rightarrow P, l=0,1, \ldots, m-1$, such that $\varphi_{l}\left(e_{(i))}=g_{l}(i)\right.$,

$\varphi_{l}(f(i))=g_{l+1}(i)$, and

$\varphi_{l}(e(k))=\varphi_{l}(f(k))=g(k)=h(k)$ for $k \neq i$.

This proves $\left(g_{l}, g_{l+1}\right) \in \operatorname{Con}(e, f)$ for all $l=0,1, \ldots, m-1$, hence $(g, h) \in$ $\in \operatorname{Con}(e, f)$. Consequently, $Z(D(e, f)) \subseteq \operatorname{Con}(e, f)$.

The last lemma is in a contradiction with our basic assumption $Z(D(e, f)) \neq$ $\neq \operatorname{Con}(e, f)$. Hence in fact $Z(D(e, f))=\operatorname{Con}(e, f)$ for all couples $(e, f) \in E \times E$. Since the set $\{\operatorname{Con}(e, f):(e, f) \in E \times E\}$ is a join-generating subset of $\operatorname{Con}(E, G)$ (see e.g. [8]), we have $\operatorname{Con}(E, G) \subseteq \operatorname{Im}(Z)$.

\section{References}

[1] G. Birkhoff: On the structure of abstract algebras, Proc. Cambridge Phil. Soc., Vol. 31 (1935), 433-454.

[2] N. Bourbaki: Groupes et algebres de Lie, Chap. 4, 5 et 6, Éléments de mathematique, Fasc. XXXIV, Hermann, Paris, 1968.

[3] R. W. Carter: Simple groups of Lie type, Wiley, London, 1972.

[4] R. McKenzie: Finite forbidden lattices, Lecture Notes in Math., No. 1004, Springer Verlag (1983), 176-205.

[5] R. McKenzie: Tame congruences, preprint.

[6] R. McKenzie: The structure of finite algebras, preprint.

[7] P. P. Pálfy and P. Pudlák: Congruence lattices of finite algebras and intervals in subgroup lattices of finite groups, Algebra Universalis, 11 (1980), 22-27.

[8] P. Pudlák: Distributivity of strongly representable lattices, Algebra Universalis, 7 (1977), $85-92$.

[9] P. Pudlák and J. Tüma: Yeast graphs and fermentation of algebraic lattices, Coll. Math. Soc. János Bolyai, 14. Lattice Theory, Szeged, 1974.

[10] J. Tits: Buildings of spherical type and finite BN-pairs, Lecture Notes in Math., No. 386, Springer Verlag (1974).

[11] J. Tits: A local approach to buildings, in "The geometric vein (The Coxeter Festschrift)", edited by C. Davis, B. Grünbaum and F. A. Sherk, Springer Verlag (1981), 519-547.

[12] P. Pudlák and $J$. Tüma: Every finite lattice can be embedded in a finite partition lattice, Algebra Universalis 10 (1980), 74-95.

[13] H. L. Silcock: Generalized wreath products and the lattice of normal subgroups of a group, Algebra Universalis, 7 (1977) 361-372.

Author's address: 11567 Praha 1, Žitná 25, Czechoslovakia (Matematický ústav ČSAV). 